\title{
Trading Genome Vulnerability for Stable Genetic Inheritance: Active Retrotransposons Help Maintain Pericentromeric Heterochromatin Required for Faithful Cell Division
}

Yajing $\mathrm{Hao}^{1,2,3,4}$, Dongpeng Wang ${ }^{1,2,4}$, Shuheng $\mathrm{Wu}^{1,2}, \mathrm{Xiao} \mathrm{Li}^{3}$, Changwei Shao ${ }^{3}$, Peng Zhang ${ }^{1}$, Jia-Yu Chen ${ }^{3}$, Do-Hwan $\mathrm{Lim}^{3}$, Xiang-Dong $\mathrm{Fu}^{3, *}$, Shunmin $\mathrm{He}^{1, *}$, and Runsheng Chen ${ }^{1, *}$

${ }^{1}$ Key Laboratory of RNA Biology, Institute of Biophysics, Chinese Academy of Sciences, 100101, China

${ }^{3}$ Department of Cellular and Molecular Medicine, University of California, San Diego, La Jolla,

23 Xiang-Dong Fu (xdfu@ucsd.edu); Shunmin He (heshunmin@ibp.ac.cn); Runsheng Chen

24 (chenrs@sun5.ibp.ac.cn) 


\section{ABSTRACT}

Retrotransposons are extensively populated in vertebrate genomes, which, when active,

27 are thought to cause genome instability with potential benefit to genome evolution.

28 Retrotransposon-derived RNAs are also known to give rise to small endo-siRNAs to help maintain

29 heterochromatin at their sites of transcription; however, as not all heterochromatic regions are

30 equally active in transcription, it remains unclear how heterochromatin is maintained across the

31 genome. Here, we attack these problems by defining the origins of repeat-derived RNAs and their

32 specific chromatin registers in Drosophila S2 cells. We demonstrate that repeat RNAs are

33 predominantly derived from active Gypsy elements, and upon their processing by Dicer-2, these

34 endo-siRNAs act in cis and trans to help maintain pericentromeric heterochromatin. Remarkably,

35 we show that synthetic repeat-derived siRNAs are sufficient to rescue Dicer-2 deficiency-induced

36 defects in heterochromatin formation in interphase and chromosome segregation during mitosis,

37 thus demonstrating that active retrotransposons are actually required for stable genetic inheritance. 


\section{INTRODUCTION}

Eukaryotic genomes contain both gene-rich and gene-poor regions, respectively corresponding to euchromatin and heterochromatin. Heterochromatin can be further divided into

41 two classes: facultative, which is dynamic and marked by H3K27me3, and constitutive, which is

42 largely stable and marked by H3K9me2/3(Grewal and Jia 2007). Constitutive heterochromatin is

43 predominately associated with centromeric and pericentromeric regions, telomeres, and

44 retrotransposons(Lippman et al. 2004). Constitutive heterochromatin plays important roles in

45 genome organization in the nucleus(Avner and Heard 2001; Zhang et al. 2019), suppression of

46 recombination to protect genome integrity(Grewal and Klar 1997), and stable genetic inheritance

47 during development and differentiation(Allshire et al. 1995; Peters et al. 2001). These critical

48 biological functions underscore the importance of repeat-rich sequences underneath constitutive

49 heterochromatin, which used to be referred to as "junk" DNA in the genome. In fact, besides their

50 potential contribution to genome evolution, it has been unclear whether active retrotransposons

51 have any immediate benefit to an organism.

53 generally referred to as heterochromatin), our current knowledge is largely derived from elegant

54 genetic and biochemical studies in fission yeast and Drosophila melanogaster(Tschiersch et al.

55 1994; Grewal and Jia 2007; Holoch and Moazed 2015b). The most striking aspect of the emerging

56 theme is that transcription is required for initiating heterochromatin formation, even though the

57 eventual fate is to shut down transcription. In fission yeast, initial repeat-derived transcripts are 
amplified by an RNA-dependent RNA polymerase (RdRP). Resultant double-stranded RNAs are

RNA. RITS recruits a key histone methyltransferase Clr4 (Su(var)3-9 in flies and SUV39H1 in

75 a pericentromeric region of one chromosome and the other in its native euchromatic context of

77 principle generally applies to heterochromatin maintenance on all pericentromeric regions, and if 
so, what is the relative contribution of cis- versus trans-acting RNAs to such maintenance. Third,

both fission and Drosophila germ cells are equipped with an RNA amplification system (RNA-

81 is lacking in somatic cells of flies and mammals(Stein et al. 2003). The question is how somatic

82 cells meet this supply/demand dilemma. Forth, fission yeast uses siRNA to drive heterochromatin

83 formation and maintenance, but fly enlists the piRNA pathway for such purpose in the germline.

84 This raises the question of whether different organisms may use distinct machineries for processing

85 repeat-derived RNAs. Last, but not least, Dicer deficiency has been reported to cause chromosome

86 mis-segregation during mitosis in both flies and mammals(Pek and Kai 2011; Huang et al. 2015),

87 but it has remained unclear whether such dramatic phenotype results from impaired production of

88 some sort of endo-siRNAs or other function(s) of Dicer in the nucleus.

Addressing the above questions would require critical information on where repeat-rich

90 transcripts are generated and where these repeat RNAs target specific loci in the genome, which

91 has been a challenging problem. Recent technological innovations have made it possible to

92 comprehensively elucidate the RNA-chromatin interactome, which has been applied to multiple

93 cell types, including Drosophila S2 cells(Li et al. 2017; Sridhar et al. 2017; Bell et al. 2018), but

94 rich information on repeat-derived RNAs has not yet been explored. We have now utilized this

95 information to define the interaction of repeat-derived RNAs with chromatin across the fly genome.

96 Our analysis reveals that endo-siRNAs are mostly derived from the Gypsy family of

97 retrotransposons, which are able to act in cis and trans to balance local transcription to help 
maintain pericentromeric heterochromatin. Strikingly, we show that a pool of synthetic endo-

99 siRNA mimics is sufficient to rescue chromosome segregation defects in Dicer-deficient cells.

100 These findings reveal that active retrotransposons are functionally required for maintaining

101 heterochromatin to ensure stable genetic inheritance during cell cycle.

102

103

\section{RESULTS}

104 Strategy for genome-wide assignment of multi-mapped RNA and DNA reads

We recently developed a technology called global RNA-DNA interaction sequencing

106 (GRID-seq) to detect chromatin-associated RNAs and their respective binding sites genome-wide.

107 GRID-seq employs a bivalent linker to ligate to RNA in one end and fragmented DNA in the other

108 end on fixed nuclei followed by selection and cleavage of linker ligated products with a type IIS

109 restriction enzyme (Mme I) to generate "mated" RNA and DNA (both 20nt in length) for deep

110 sequencing(Li et al. 2017). To control for the specificity in RNA-DNA mating, we also generated

111 a GRID-seq library on mixed human and fly cells, thus enabling the construction of a critical

112 background model by using cross-species reads (i.e. fly RNA ligated to human DNA and vice

113 versa). Using these datasets, we previously explored uniquely mapped RNA-DNA mates to reveal

114 nascent RNA-covered "transcription hubs" where specific promoters and enhancers are

115 interconnected in the nucleus(Li et al. 2017). Given such high-quality data, we herein explored the

116 biological significance of multi-mapped RNA and DNA reads in Drosophila S2 cells, taking

117 advantage of high density reads on the much smaller genome of fly relative to humans. 
A

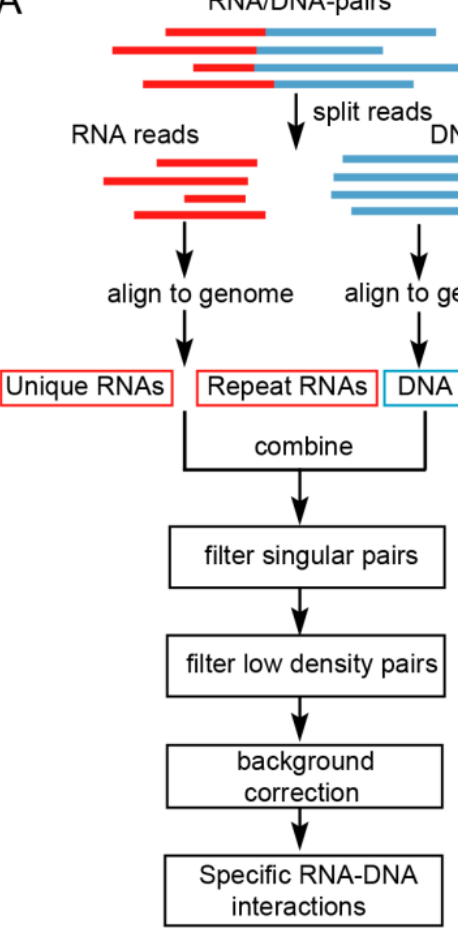

B

DNA interaction with unique RNA

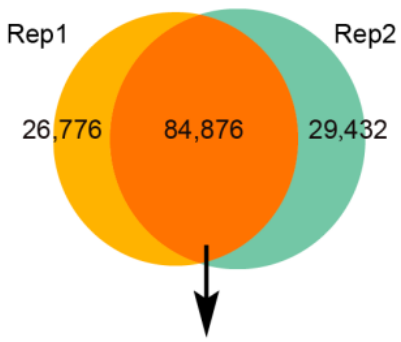

Frequency of RNA-DNA contacts $\left(\log _{2}(\mathrm{RRPKM})\right)$

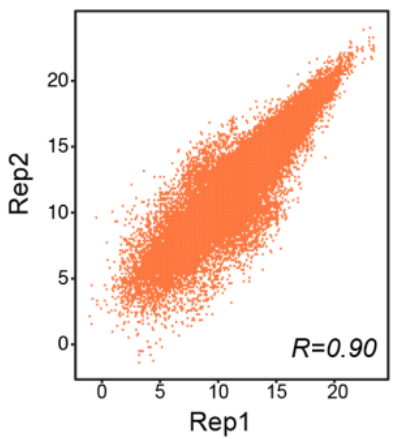

G
C

DNA interaction with repeat-derived RNA

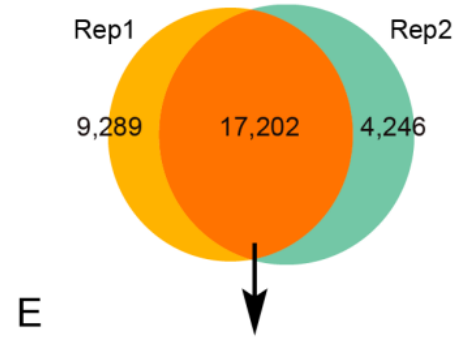

Frequency of RNA-DNA contacts $\left(\log _{2}(\right.$ RRPKM $\left.)\right)$

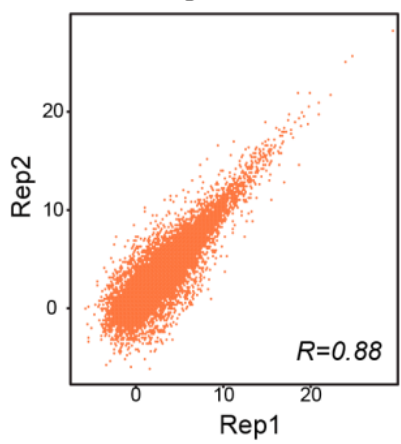

DNA
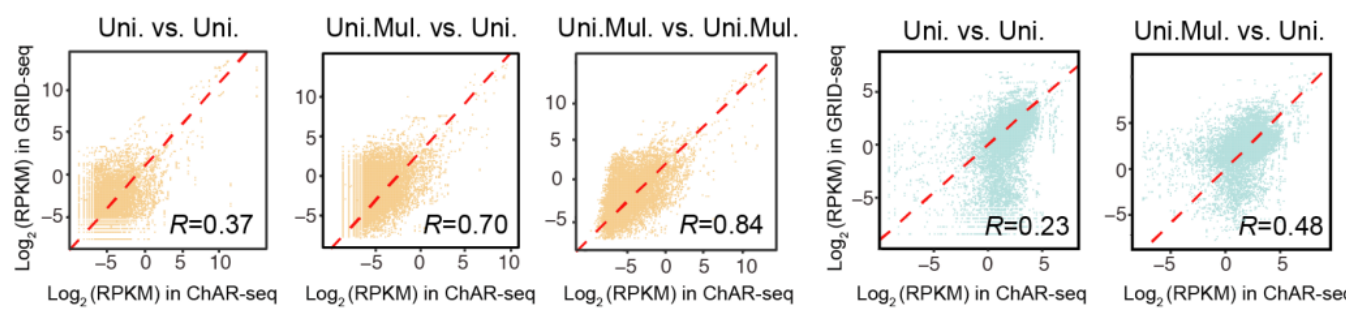

Uni.Mul. vs. Uni.Mul

$\mathrm{H}$

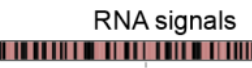
chr3L(78F1-79F 2$)$

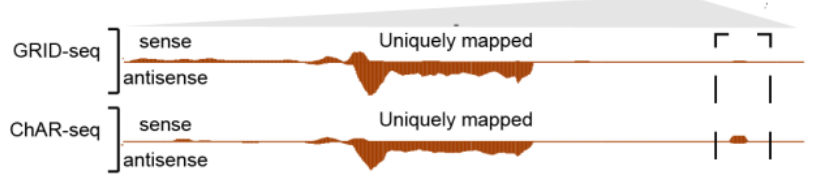

I DNA signals

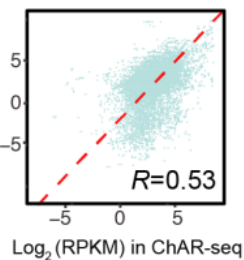

$\log _{2}\left(\right.$ RPKM) in ChAR-seq $\log _{2}$ (RPKM) in ChAR-seq

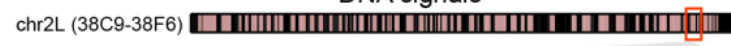

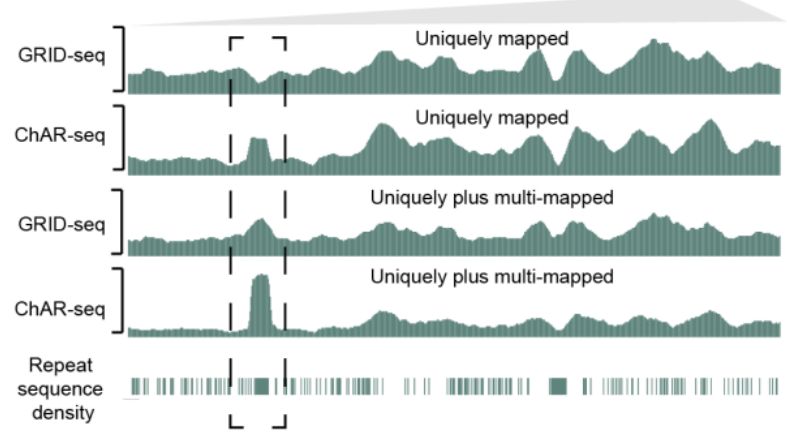

GRID-seq $]$ sense Uniquely plus multi-mapped

ChAR-seq $]$ sense Uniquely plus multi-mapped

Repeat

sequence

|

density

ᄂ $\lrcorner$

Figure 1. Strategy for assigning multi-mapped RNA and DNA reads

120 (A) Schematic presentation of the GRID-seq data processing pipeline. (B and C) Overlapped

121 RNA-DNA mates containing annotated unique (B) or repeat-derived (C) RNA between the two

122 independent GRID-seq libraries.(D and E) Quantitative analysis of commonly identified RNA- 
123 DNA mates containing annotated unique RNA (D) or repeat-derived RNA (E) between the two 124 independent GRID-seq libraries. RRPKM: Read counts per kilobase of RNA per kilobase of DNA 125 per million. ( $F$ and $G$ ) Comparison between GRID-seq and ChAR-seq with uniquely or uniquely 126 plus multi-mapped RNA (F) or DNA (G) in repeat-enriched Alu I-generated DNA bins. Uni.: 127 uniquely mapped reads. Uni.Mul.: uniquely plus multi-mapped reads. (H and I) Transcribed 128 RNA signals $(\mathrm{H})$ or RNA-contacted DNA signals (I) obtained with different mapping strategies 129 on representative genomic regions.

130 The two independently generated GRID-seq libraries on S2 cells (Supplementary Table

131 1) were of high global concordance between RNA or DNA reads (Supplementary Fig. 1A).

132 Interestingly, both replicates showed a high percentage (65\% to $75 \%$ ) of RNA reads that were

133 mapped to repeat-derived transcripts, but a much smaller fraction (10\% to 20\%) of DNA reads

134 were assigned to repeat-rich genomic loci (Supplementary Fig. 1B). To fully utilize these multi-

135 mapped RNA and DNA reads, we took a previously established ShortStack strategy to make

136 assignment to specific transcripts and genomic locations based on the local density of uniquely

137 mapped reads(Axtell 2013). As illustrated (Fig. 1A), we first aligned each RNA read to genome

138 and assigned it to annotated unique RNA transcripts according to the FlyBase database(Drysdale

139 2008) or repeat-derived transcripts based on the RepeatMasker database

140 (http://www.repeatmasker.org), and each DNA read to genomic fragments(Roberts et al. 2015),

141 generated by Alu I, a restriction enzyme used to fragment the fly genome during library

142 construction. For multi-mapped reads, we distributed them to individual RNA transcripts or DNA

143 fragments according to the relative density of uniquely mapped reads (Supplementary Fig. 1C).

145 threshold according to the Poisson distribution of all mates across the genome (see Online 
146 METHODS). Additionally, we also subtracted the background based on human RNA signals

147 mapped to fly DNA loci from our fly/human GRID-seq library. The density of such background

148 reads is quite significant in many accessible chromatin regions (Supplementary Fig. 1D), as

149 detailed earlier(Li et al. 2017). After these data processing steps, retained RNA-DNA mates show

150 high consistency between the two independent GRID-seq libraries, as indicated by predominant

151 common mates associated with both annotated unique RNAs (Fig. 1B) and repeat-derived

152 transcripts (Fig. 1C). This consistency is also reflected at the quantitative levels of individual

153 common RNA-DNA mates (Fig. 1D,1E), thus enabling us to rely on these common mates to

154 generate the final RNA-DNA interactome for downstream analysis. Notably, after assigning multi-

155 mapped RNA and DNA reads, most gaps around repeat-rich DNA regions were "filled" to the

156 similar levels, as compared to adjacent unique regions across the fly genome (Supplementary Fig.

$1572 \mathrm{~A})$.

159 Validation of mapping results with an independent dataset

160 It is critical to validate our mapping strategy, even though ShortStack has been generally

161 accepted as a strategy to dynamically assign multi-mapped reads in a given genome. For this

162 purpose, we utilized the data generated by ChAR-seq, a strategy similar to GRID-seq except longer

163 RNA and DNA reads were generated by sonication after linker ligation (Supplementary Fig.

164 2B)(Bell et al. 2018). Compared to GRID-seq that generates predominantly mated RNA-DNA

165 pairs, ChAR-seq tends to trade off relative longer reads with a large fraction of unmated RNA or 

where many other types of genomic data are available for comparison (see Supplementary Table 2), whereas ChAR-seq libraries were produced on a less commonly used Drosophila cell line (CME-

171 Table 1), thus prohibitive to assessing internal data reproducibility, and most importantly, (iii) one

172 of our GRID-seq libraries was constructed on mixed fly and human cells, thus permitting the use

173 of cross-species RNA-DNA mates to build a background for non-specific RNA-DNA interactions,

174 which is missing from the existing ChAR-seq libraries. Nevertheless, the available ChAR-seq data

175 with longer RNA and DNA reads provided an independent dataset to evaluate our strategy for

176 assigning multi-mapped RNA and DNA reads, despite the fact that GRID-seq and ChAR-seq

177 libraries were derived from different fly cell types. We first compared between ChAR-seq and GRID-seq data, observing an overall high

179 Spearman correlation ( $\mathrm{R}=0.75$ for $\mathrm{RNA}$ reads; $\mathrm{R}=0.62$ for $\mathrm{DNA}$ reads) across the reference fly

180 genome (Supplementary Fig. 2C). However, when focused on repeat-enriched Alu I-generated

181 DNA bins, the correlation was quite modest at the levels of both RNA (left panel in Fig. 1F, R=0.37)

182 and DNA (left panel in Fig. 1G, R=0.23). Notably, a population of DNA reads (distributed in lower

183 right in Fig. 1G) was scored by ChAR-seq, but less by GRID-seq, likely due to the higher mapping

184 power of the former. Interestingly, the correlation was dramatically improved when comparing 185 uniquely mapped RNA or DNA reads from ChAR-seq with uniquely plus multi-mapped RNA or 
197 visible after assigning multi-mapped GRID-seq reads. Together, these data validate our

198 computational strategy to assign multi-mapped RNA and DNA reads to the genome.

\section{Preferential interaction of distinct RNA classes with eu- versus hetero-chromatin}


211 RNA species, and thus, only those with sufficient expression were detectable on chromatin.

A

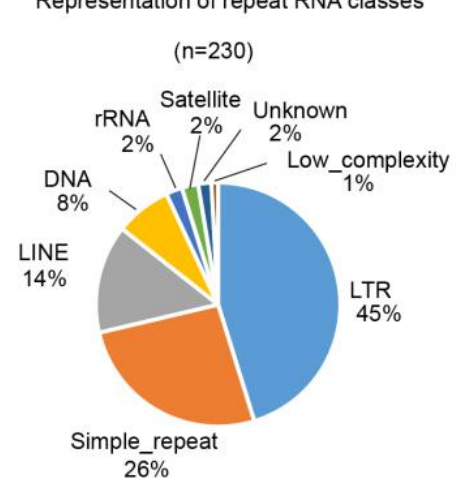

C

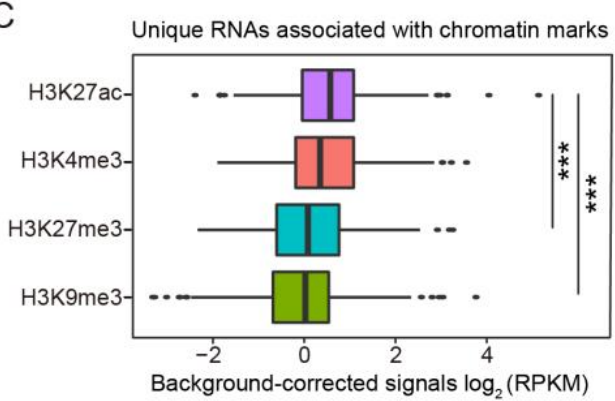

D

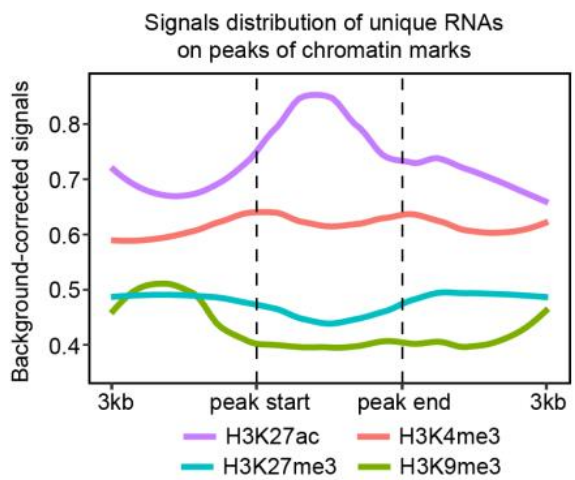

B

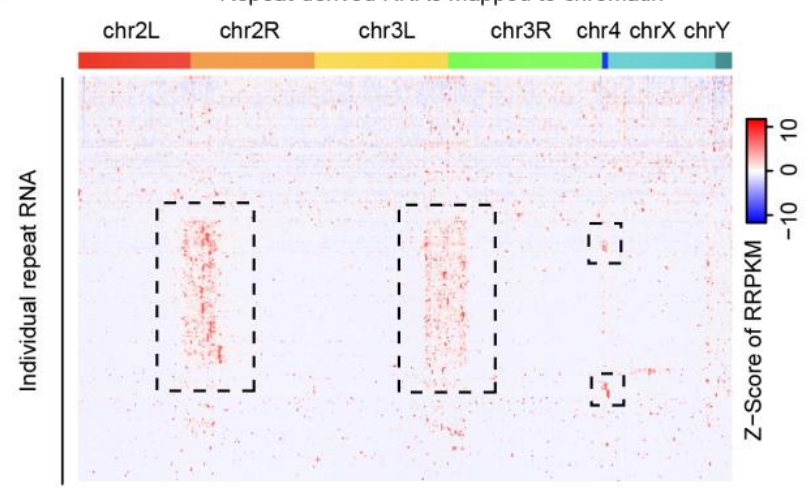

E

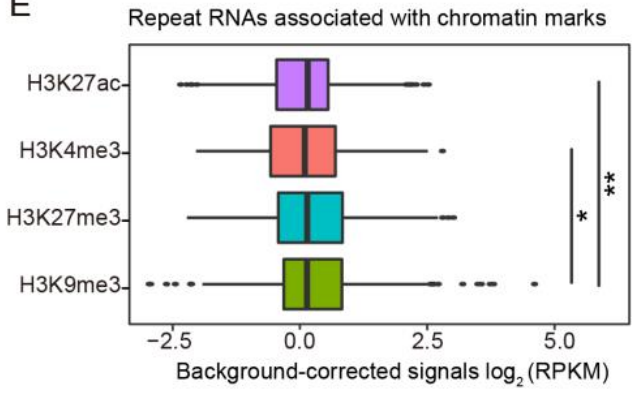

F

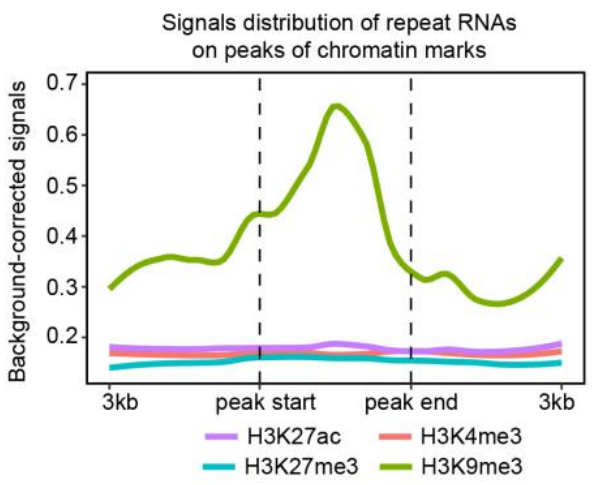




\section{Figure 2. Interaction of distinct RNA classes with eu- versus hetero-chromatin}

214 (A) Different repeat RNA classes represented by 230 DNA bound repeat-derived RNAs. (B)

215 Heatmap showing the distribution of individual 230 repeat-derived RNAs across the Drosophila

216 genome in S2 cells. Row: Individual chromatin-associated repeat-derived RNAs. Column: AluI

217 DNA bins. Boxed regions: Repeat-derived RNAs that showed preferential binding to constitutive

218 heterochromatin in pericentromeric regions. (C) Association of chromatin marks with background219 corrected signals of unique RNAs. ${ }^{*} \mathrm{p}<0.05$, ${ }^{*} \mathrm{p}<<0.01$, $* * * \mathrm{p}<0.001$ (unpaired Student's t-test).

220 (D) Distribution of unique RNA interaction signals around chromatin mark peaks. (E) Association 221 of chromatin marks with background-corrected signals of repeat-derived RNAs. *p<0.05, $222 * * \mathrm{p}<0.01$ (unpaired Student's t-test). (F) Distribution of repeat RNA interaction signals around 223 chromatin mark peaks.

identified 230 repeat-derived RNA species that showed significant interactions with chromatin,

which include all rRNAs, satellite DNA-transcribed RNAs (including those from simple repeats), 
Fig. 3D). Together, these data suggest a general trend in which annotated non-repeat RNAs tend

more prevalent than others in interacting with specific heterochromatic regions in the fly genome.

246 This established the critical foundation to investigate their relative contributions to the initiation

247 and/or maintenance of heterochromatin in Drosophila cells.

\section{Prevalent association of Gypsy-derived RNAs with constitutive heterochromatin}

252 constitutive heterochromatin characterized by the coordinated ChIP-seq signals for H3K9me3 and

253 its reader HP1 (Fig. 3A). By determining the co-localization coefficient for each of the 230 repeat-

254 derived RNAs between their DNA interaction frequencies and relative densities of $\mathrm{H} 3 \mathrm{~K} 9 \mathrm{me} 3$ and

255 HP1 signals in 1Mb DNA bins of the fly genome, we identified 79 repeat-derived RNAs, including

256 two rRNAs, that showed the Pearson correlation coefficient of $>0.3$ (red dots in Fig. 3A). The 
258 inert centromere-proximal regions tend to be organized around the nucleolus in 3D

259 genome(Quinodoz et al. 2017). However, since rRNAs are assembled into ribosomes, rather than

260 processed into small RNAs, it is unlikely that they contribute to heterochromatin functions.

261 Excluding rRNAs, we named the rest of heterochromatin-enriched RNAs as CHARRs

262 (으stitutive $\underline{H}$ eterochromatin- $\underline{\text { Associated }}$ Repeats-derived $\underline{\text { RNAs}}$ ). These CHARRs appear to

263 show exclusive association with constitutive heterochromatin, as none of them exhibited

264 significant co-localization with the facultative chromatin marker H3K27me3 (Supplementary Fig.

265 4A), as illustrated with three specific CHARRs (Supplementary Fig. 4B). Furthermore, these

266 CHARRs showed little association with MSL (Supplementary Fig. 4C,D), a key component of the

267 silencing complex involved in $\mathrm{X}$ inactivation in Drosophila, consistent with the nature of

268 predominant facultative heterochromatin formed on X-inactivation (Xi)(Baker et al. 1994; Franke

269 and Baker 1999). 
A
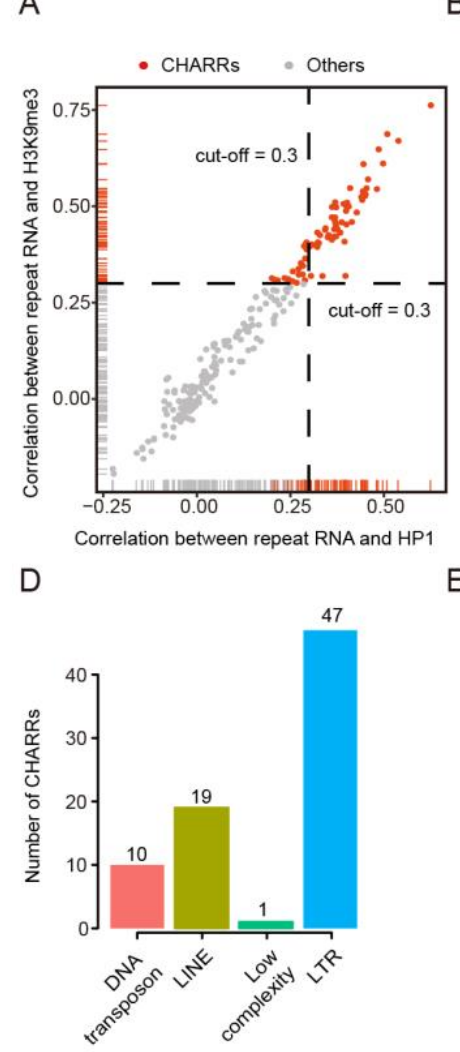

B
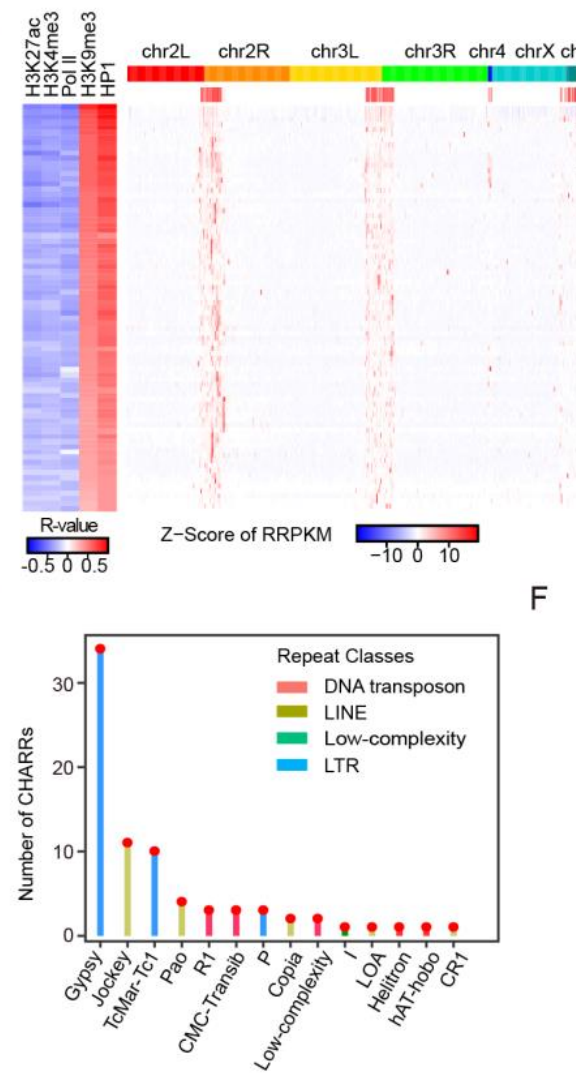

C

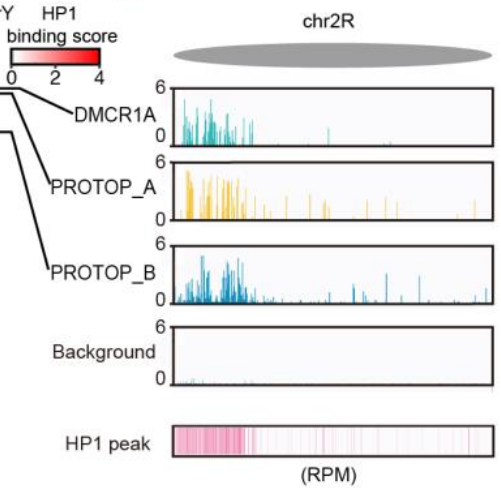

$\mathrm{F}$

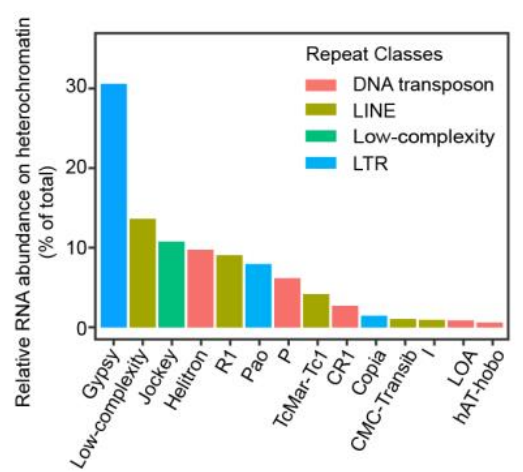

\section{Figure 3. Prevalent association of Gypsy-derived RNAs with constitutive heterochromatin}

(A) Scatterplot of co-localization coefficients between repeat-derived RNA signals on DNA and the levels of H3K9me3 (y-axis) or HP1 (x-axis) in S2 cells. A threshold of 0.3 was chosen for both chromatin marks (red lines). Dashed lines were used to differentiate CHARRs (red dots) from other repeat-derived RNAs (grey dots). (B) Left: Heatmap of co-localization of each CHARR with individual chromatin marks or Pol II ChIP-seq signals. Right: Heatmap of chromatin interaction of each CHARR with Alu I-generated DNA bins, which were merged from 100 continuous Alu I DNA bins. HP1 binding signals in these DNA bins are displayed below the schematic presentation of Drosophila chromosomes. Three representative CHARRs are labeled on the right. (C) Chromatin interaction signals of three representative CHARRs, DMCR1A, PROTOP_A and PROTOP_B on a genomic region in chromosome $2 \mathrm{R}$ in comparison with non-specific background based on human RNA mapped to fly DNA and HP1 binding density. All signals were scaled to reads per million. (D and E) The number of repeat classes (D) or sub-families (E) associated with CHARRs. (F) The relative RNA abundance (\% of total) of CHARRs on constitutive heterochromatin and to. 
294 and relative abundance for each CHARR. Interestingly, the largest RNA class of identified

CHARRs corresponds to LTR $(n=47)$, while the second largest class to LINE repeats $(n=19)($ Fig.

3D). The majority of RNA species from these two classes of retrotransposons belongs to the Gypsy contributors to the overall RNA signals on heterochromatin (Fig. 3F). This suggests a major role of Gypsy-derived RNAs in heterochromatin formation/maintenance in Drosophila $\mathrm{S} 2$ cells.

\section{Cis- and trans-acting repeat-derived RNAs on chromatin}


mapped to different chromosomes), we asked whether this also applied to repeat-derived RNAs.

309 We noted that rRNA-derived RNAs all interacted with heterochromatin regions near the loci of

310 their transcription where CHARRs also predominantly bound (Supplementary Fig. 5A,B). This

311 suggests multiple active rRNA transcription sites are in close spatial proximity with adjacent

312 pericentromeric regions, as recently observed based on proximity ligation(Quinodoz et al. 2017).

313 Importantly, we also identified CHARRs on multiple non-pericentromeric regions, suggesting

314 their potential interactions with chromatin in both cis- and trans-modes.

We therefore determined the origins of CHARRs and their collective interactions with

316 chromatin at the chromosomal levels. This analysis revealed their extensive chromatin interactions

317 not only within the same chromosomes, but also across different chromosomes (Fig. 4A),

318 suggesting a significant degree of trans-interactions. This prompted us to examine individual

319 CHARRs to segregate their cis- and trans-interactions by first assigning their origins of

320 transcription, and then determining their linkage to DNA on the same (intra) or different (inter)

321 chromosomes. Interestingly, we found that about half (46.8\%) of the CHARRs were

322 predominantly engaged in intra-chromosomal interactions, whereas the other half $(53.2 \%)$ were

323 actively involved in both intra- and inter-chromosomal interactions (Fig. 4B). This is further

324 illustrated with 3 representative CHARRs. Specifically, Gypsy12_LTR preferentially interacted

325 with DNA on the same chromosome (Fig. 4C); Gypsy4_I-int was engaged in both intra- and inter-

326 chromosomal interactions (Fig. 4D); and FW_DM seemed to mainly act in trans on other 
bioRxiv preprint doi: https://doi.org/10.1101/740753; this version posted August 20, 2019. The copyright holder for this preprint (which was not certified by peer review) is the author/funder, who has granted bioRxiv a license to display the preprint in perpetuity. It is made available under aCC-BY-NC-ND 4.0 International license.

327 chromosomes (Fig. 4E). It is also interesting to note that individual CHARRs all selectively bound

328 Hi-C defined "B" domains in pericentromeric regions (Supplementary Fig. 5C,D).

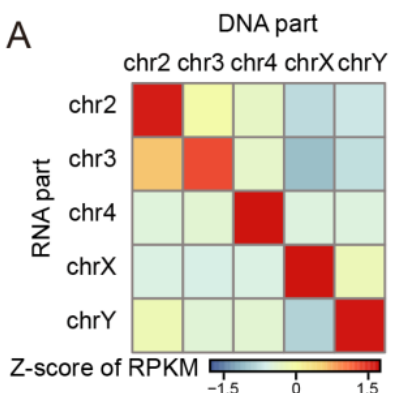

C
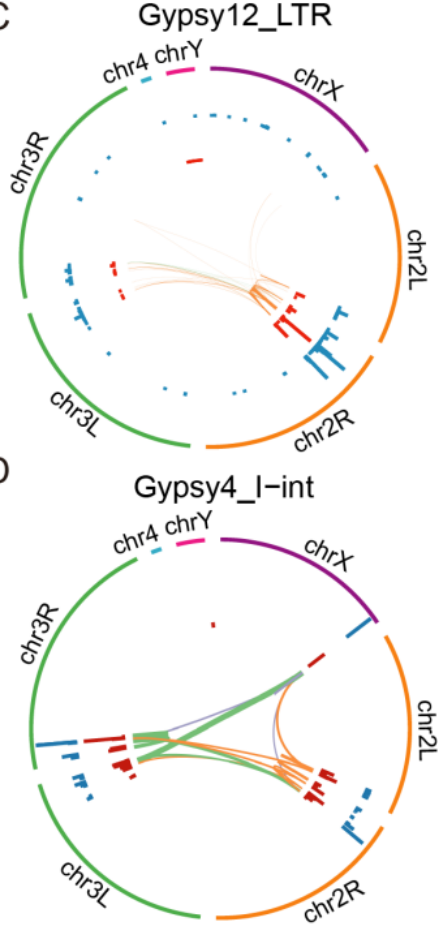

$\mathrm{E}$

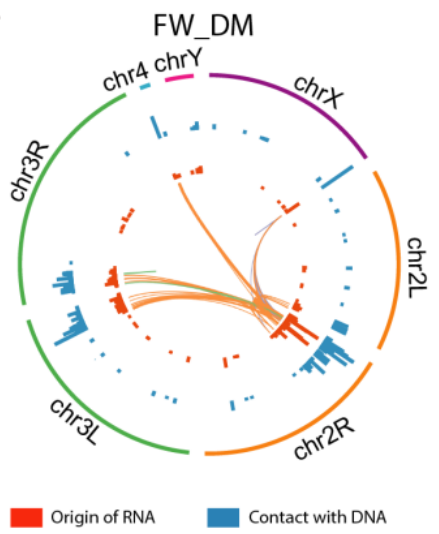

B

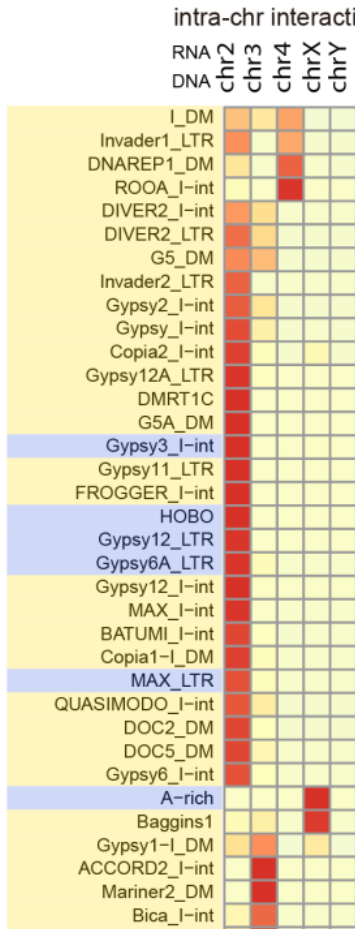

Bica_l-int G4_DM

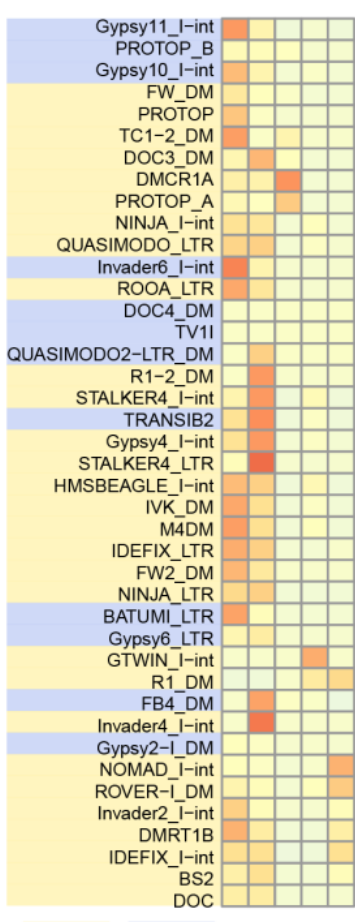

Homo inter-chr interactions

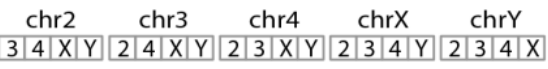
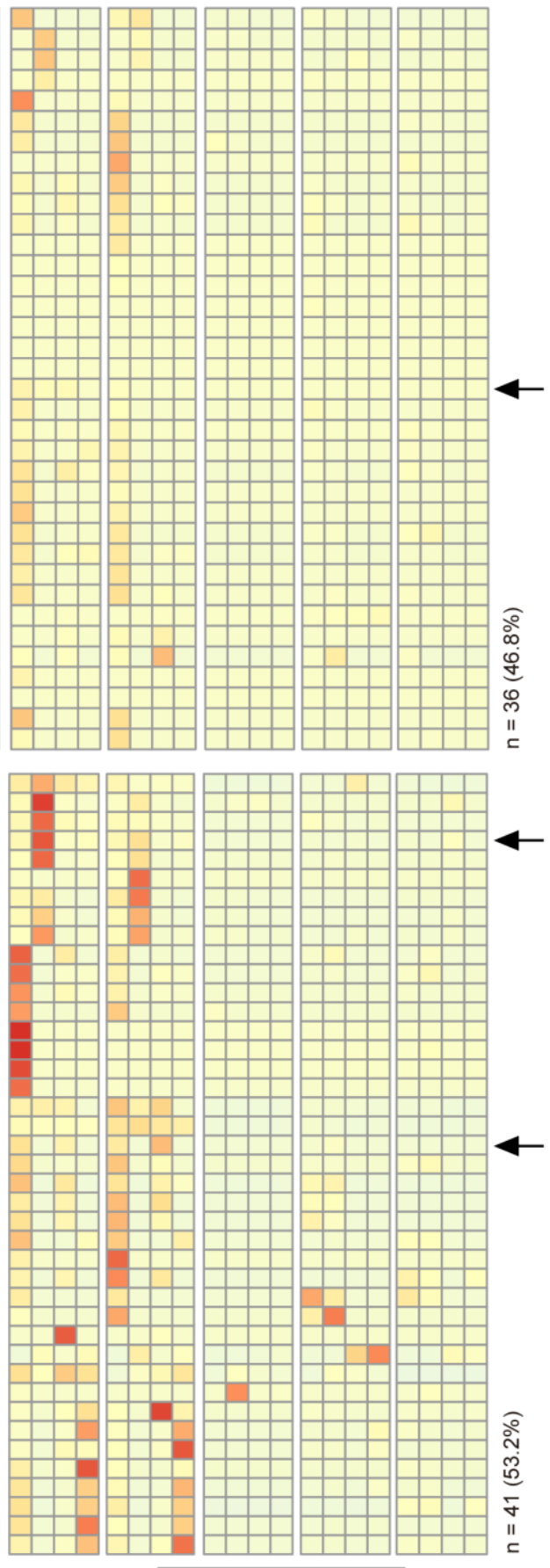

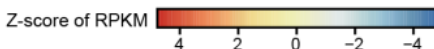




\section{Figure 4. Cis- and trans-acting repeat-derived RNAs on chromatin}

332 (A) Heatmap of RNA-DNA interaction scores for 77 CHARRs on the same or different 333 chromosomes. Trans-chromosome interactions are referred to as RNA interacts with DNA in 334 chromosomes other the RNA is transcribed from. (B) Assignment of each of the 77 CHARRs to 335 either intra- or inter-chromosomal interactions in S2 cells and sorting of the data by unsupervised 336 clustering. Top: RNA derived from different chromosomes (first row) and its DNA contacts in the 337 same or different chromosomes (second row). The RNA-DNA interaction intensities were 338 indicated by the z-scores according to the color key at the bottom. Arrows point to the three specific 339 CHARRs Gypsy12_LTR, Gypsy4_I-int and FW_DM, as individually illustrated in panel C, D, 340 and E. All CHARRs are either highlighted with yellow or blue to indicate their preferences for 341 homotropic or heterotropic interactions as described later. (C, D, E) The origins of three 342 representative CHARRs and their DNA contacts, as shown by the Circos plots for Gypsy12_LTR 343 (C), Gypsy4_I-int (D) and FW_DM (E). In each of these plots, the inner (red) track indicates the 344 origins of individual RNAs and the outer (blue) track shows where the RNAs interact with DNA 345 in 100kb DNA windows. The heights of the signals correspond to Reads per 100kb window per 346 millions. Lines specify intra- or inter-chromosomal interactions with width indicating the relative 347 interaction levels in each case. The line color indicates the origin of chromatin-interacting RNAs.

Tendency for trans-acting RNAs to supplement cis-acting RNAs on chromatin During our analysis of cis- and trans-acting CHARRs, we further noted an interesting phenomenon where DNA loci expressing high levels of CHARRs tended to associate with less trans-acting RNAs (related repeat-derived RNA species transcribed from other chromosomes) and RNA (a Gypsy family member from the LTR class) was mainly transcribed from region 1 (Fig. 5A, second track), most of which contacted DNA locally around the transcribing locus (Fig. 5A,

356 third track). Interestingly, trans-acting RNAs (transcribed from TV1I-related repeat species from 
358 where a much lower level of the TV1I RNA was transcribed compared to region 1 (Fig. 5A, third

359 track). We quantified these results by dividing all active TV1I loci into 3 groups according to their

360 levels of transcription (bottom 25\%, middle 50\%, and top 25\%, Fig. 5B), and then determined the

361 ratio of trans-acting over cis- plus trans-acting RNAs for each group and observed a reverse

362 correlation between local RNA production and the percentage of association with trans-acting

363 RNAs (bars, Fig. 5B). This phenomenon also applied to another repeat RNA, Baggins1 (a LOA

364 family member from the LINE class) (Fig. 5C, D). To determine whether this represents a general

365 trend, we extended the analysis to all CHARRs and found that, with a few exceptions, most

366 followed this rule (Fig. 5E). Together, these observations suggest that highly transcribed CHARRs

367 supply RNAs in trans to interact with DNA regions with less transcription activities. 
A

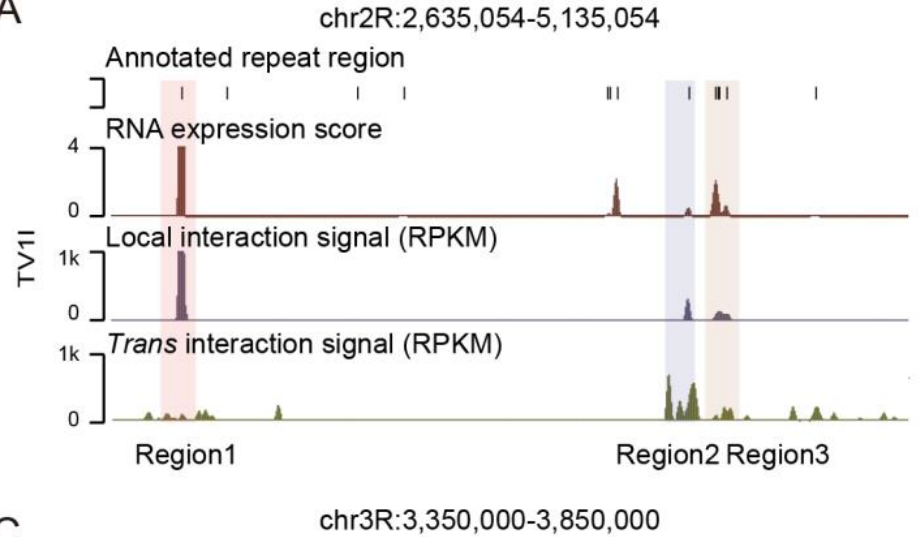

C

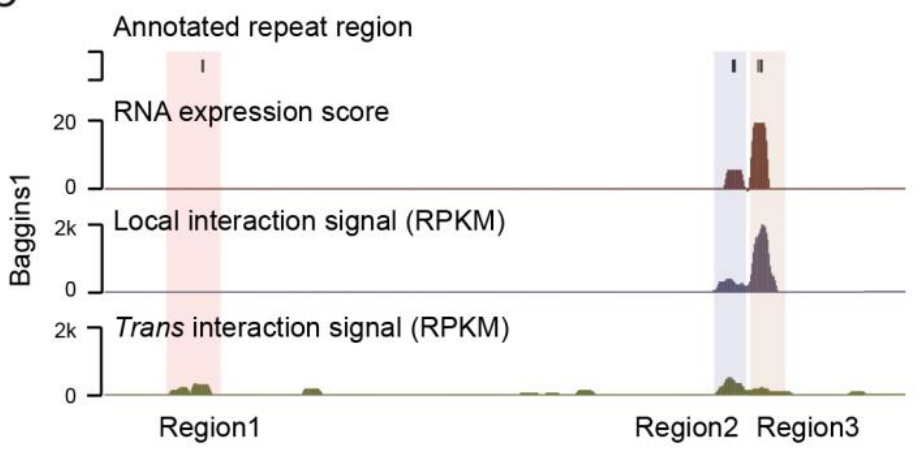

$E$

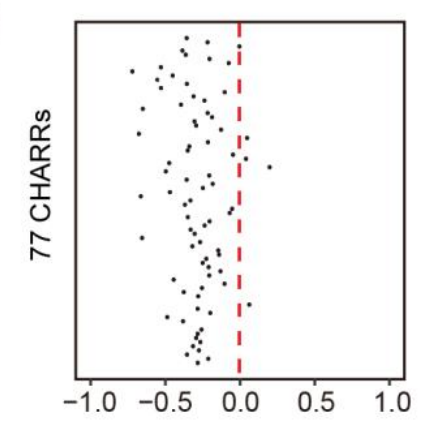

Correlation between RNA expression and \% trans-actions

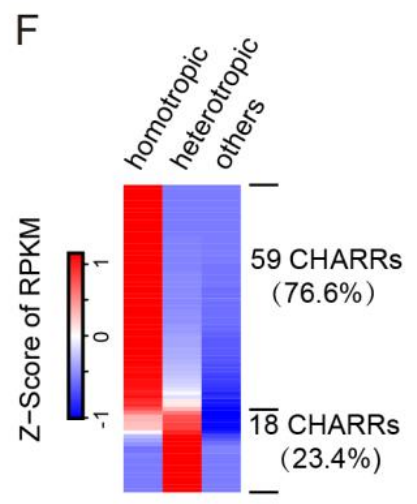

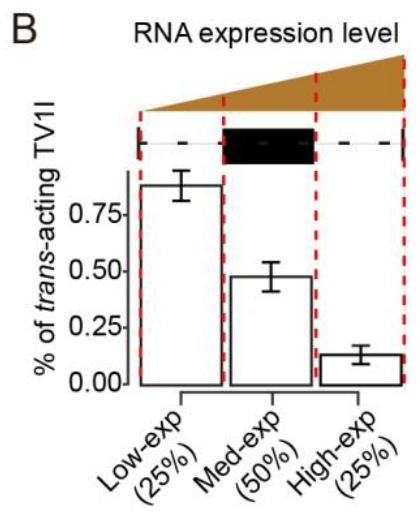

D RNA expression level

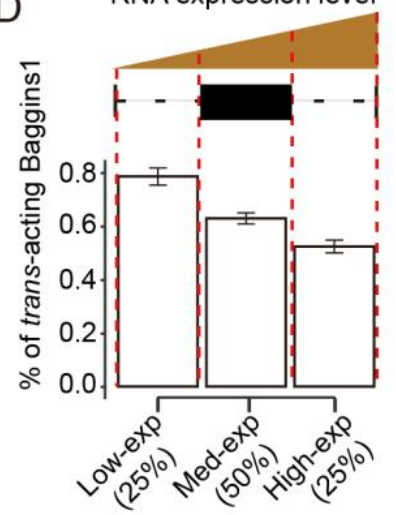

Preference for intra-chr interactions Preference for inter-chr interactions Odds ratio $=\frac{(30 / 29)}{(6 / 12)}=2.07$

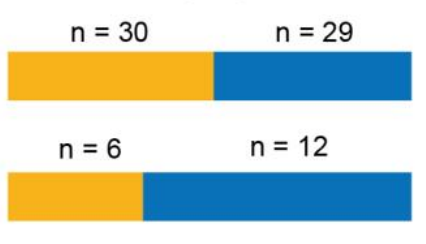

Figure 5. Reverse correlation between local transcription and association with trans-acting RNAs on chromatin

(A) A representative genomic region showing TV1I transcription loci and interactions with TV1I subfamily RNAs produced either locally or from other chromosomes (trans-acting RNAs). Three annotated TV1I transcription regions are indicated at bottom. (B) TV1I subfamily-derived RNAs were segregated into three groups according to their levels of transcription (bottom 25\%, middle $50 \%$, and top 25\%). Bars indicate the percentage of trans-acting TV1I RNAs on the transcription loci in each group. (C and D) Similar analysis and illustration for another typical CHARR Baggins 1 as in A and B.(E) Pearson correlation scores for individual CHARRs between their expression 
378 and the percentage of associated trans-acting RNAs from the same subfamilies.(F) Tendencies of 379 CHARRs engaging homotropic versus heterotropic interactions based on normalized reads per 380 kilobase per million (Left). The two classes of CHARRs were further separated into those with 381 preference for inter- (yellow) or intra- (blue) chromosomal interactions (Right), as indicated in Fig. 3824.

384 of transcription while others showed both cis- and trans-chromosomal interactions, we next

385 characterized the underlying DNA sequences that might specify such RNA-DNA interactions. If

386 a given CHARR specifically interacted with a DNA region that harbors the same repeat sequence

387 within a $1 \mathrm{~kb}$ window, we called it "homotropic" interactions. If the interacting DNA region

388 contains distinct repeat sequence (e.g. those encoding for RNAs of different classes or different

389 subfamily members), we then referred it to as "heterotropic" interactions. All DNA interaction

390 regions that contain no repeat sequence were classified as "others". According to these definitions,

391 we found that 59 CHARRs (yellow-labeled in Fig. 4B) were predominantly engaged in homotropic

392 interactions, whereas the remaining 18 (blue-labeled in Fig. 4B) were more involved in

393 heterotropic interactions, and none showed significant interactions with DNA that contain no

394 repeat sequence (Fig. 5F, left). We further noted that relative to CHARRs with preference for

395 homotropic interactions, more CHARRs with higher tendency to engage in heterotropic

396 interactions were more involved in inter-chromosomal interactions, as indicated by a significant

397 odds ratio (Fig. 5F, right). These observations imply that CHARRs with preference for homotropic

398 interactions may facilitate initiating heterochromatin formation, whereas those for heterotropic

399 interactions may play more important roles in heterochromatin spreading as well as maintenance. 


\section{Dicer-2 processed repeat-derived RNAs for heterochromatin maintenance} took advantage of the existing GRO-seq data on Drosophila S2 cells (Supplementary Table 2), showing that both sense and anti-sense repeat-derived transcripts were indeed represented in relatively equal abundance, with rRNAs served as control for predominantly sense transcription (Supplementary Fig. 6A). Interestingly, but not necessarily surprisingly, the identified CHARRs were among the most abundant repeat-derived RNAs. This was true regardless of different RNA

408 classes (Supplementary Fig. 6B), which is in contrast to annotated non-repeat transcripts, the of some long intergenic non-coding RNAs (Supplementary Fig. 6D).

412 processing into endo-siRNAs to function in heterochromatin formation/maintenance, as

413 demonstrated earlier(Fagegaltier et al. 2009; Volpe and Martienssen 2011). Furthermore, in

414 contrast to piRNA-mediated heterochromatin formation in germline, Dicer-2 has been reported to

415 be specifically devoted to endo-siRNA processing in fly somatic cells(Lee et al. 2004; Pham et al.

416 2004; Czech et al. 2008; Ghildiyal et al. 2008). To determine whether the CHARRs we identified

417 all depended on Dicer-2 for their efficient processing and thus expression, we took advantage of

418 the existing small RNA-seq data (Supplementary Table 2) to compare their expression levels

419 between wild-type, Dicer-2 knockout, and Dicer-2 rescued S2 cells(Kandasamy and Fukunaga 
421 and rescued in Dicer-2 re-expressed cells (Supplementary Fig. 7A). Note that much higher

422 expression of those endo-siRNAs in Dicer-2 re-expressed cells likely resulted from Dicer-2

423 overexpression. Importantly, the RNA-seq reads of total CHARRs from wild-type S2 cells were

424 distributed between 18 to $25 \mathrm{nt}$ in length, consistent with their processing into endo-siRNAs

425 (Supplementary Fig. 7B). These endo-siRNAs appeared to have assembled into Ago2-containing

426 complexes, as $54(75 \%)$ CHARRs could be identified in the published Ago2 RIP data

427 (Supplementary Fig. 7C). Therefore, re-analysis of these published data on CHARRs strongly

428 suggests that Dicer-2 is responsible for processing CHARRs into endo-siRNAs to help maintain

429 heterochromatin in S2 cells.

431 (Supplementary Fig. 7D, E) and confirmed drastic reduction of small RNAs derived from

432 DMCR1A or DOC by Northern blotting (Fig. 6A). Because many CHARRs were able to supply

433 RNAs in trans, as we documented in the present study, we asked whether or not the associated

434 phenotype previously detected on heterochromatin could be "rescued" with small RNAs derived

435 from CHARRs. For this purpose, we chemically synthesized a pool of endo-siRNA mimics based

436 on a representative subset of CHARRs (DMCR1A, FB4_DM, FB_DM, Gypsy4_I-inU, DOC,

437 Gypsy2-I and I_DM, Supplementary Table 3) and transfected this pool into S2 cells depleted of

438 Dicer-2. We found that relative to wild-type cells, Dicer-2 knockdown reduced H3K9me3 as

439 expected, and importantly, the CHARR-derived siRNA pool, but not scrambled siRNA, effectively 
440 restored this heterochromatin marker, while the levels of $\mathrm{H} 3 \mathrm{~K} 27 \mathrm{me} 3$ and $\mathrm{H} 3 \mathrm{~K} 4 \mathrm{me} 3$ remained

441 constant under these experimental conditions (Fig. 6B, quantified below based on triplicated

442 experiments). This was also evident at the immunocytochemical level by staining for $\mathrm{H} 3 \mathrm{~K} 9 \mathrm{me} 3$

443 (Fig. 6C). These data suggest that the CHARR-derived siRNA mimics were able to rescue

444 heterochromatin defects upon transfection (thus only acting in trans) into Dicer-2 deficient S2

445 cells. 
A

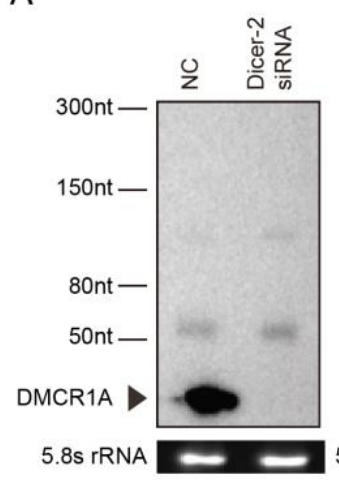

B

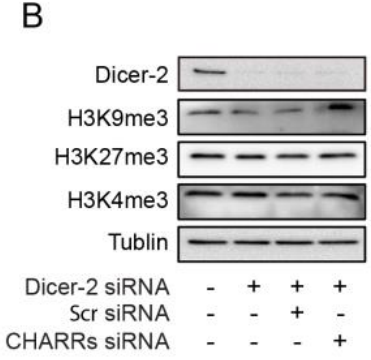

CHARRS SIRNA $\quad-\quad-\quad+\quad+$

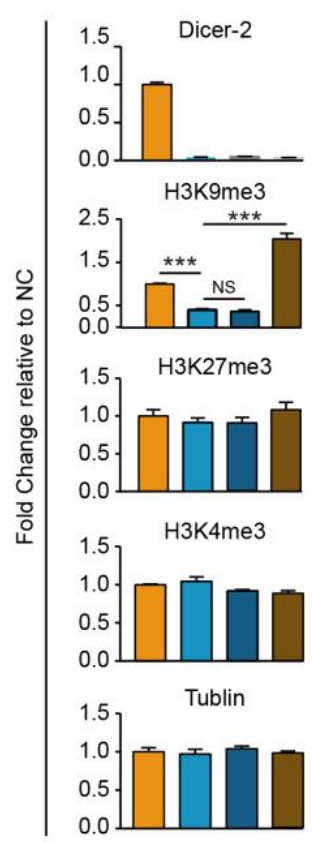

C

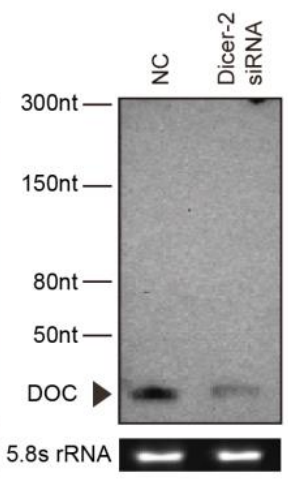

D

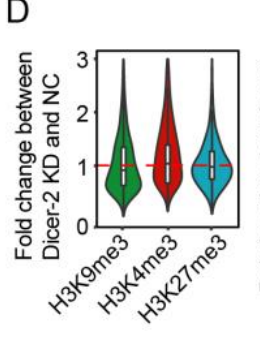

E

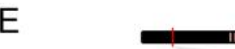

$\mathrm{F}$
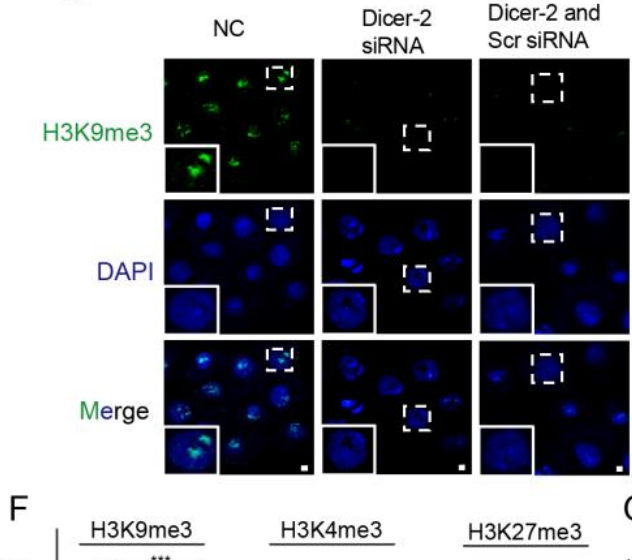

Dicer-2 an
Scr siRNA

Dicer-2 and

CHARRs siRNA
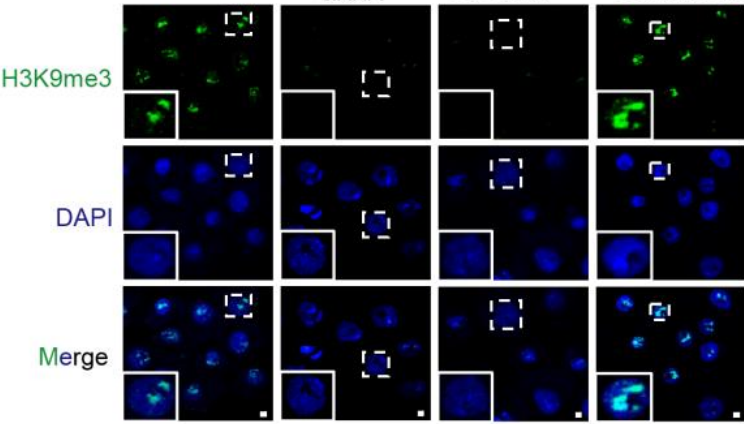

$1-1$
$i-1$

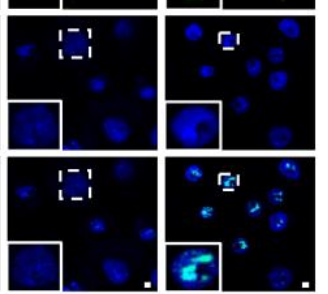

G

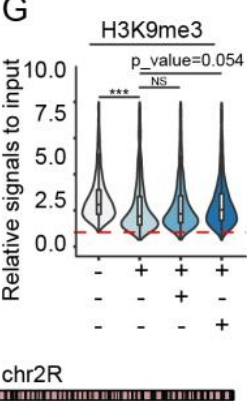

CHARRs target regions

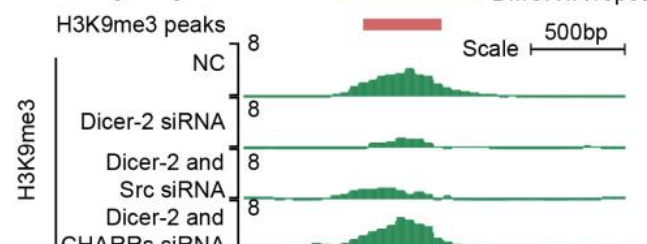

CHARRs SiRNA

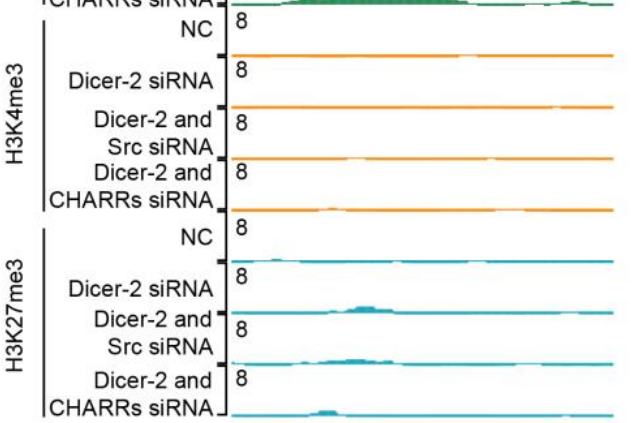

chr2R : $1,580,500-1,582,500$

ChIP-seq signals - input signals

\section{Figure 6. Trans-acting repeat-derived RNAs for heterochromatin maintenance}

448 (A) Confirmation of Dicer-2 dependent expression of DMCR1A (left) and DOC (right) expression

449 by Northern blotting analysis.(B) Western blotting analysis of Dicer-2, H3K9me3, H3K27me3,

$450 \mathrm{H} 3 \mathrm{~K} 4 \mathrm{me} 3$ and Tubulin in S2 cells in response to siRNA-mediated knockdown of Dicer-2 and

451 rescue with a transfected pool of CHARR-derived synthetic siRNAs. Quantified data are shown

452 below as fold-change (FC) relative to input (NC) in lane 1. Data are presented as mean \pm SEM ( $n$ 
$453=3$ biological replicates). ${ }^{*} \mathrm{p}<0.05, * * \mathrm{p}<0.01, * * * \mathrm{p}<0.001$. (unpaired Student's $\mathrm{t}$ test).(C)

$454 \mathrm{H} 3 \mathrm{~K} 9 \mathrm{me} 3$ detected by immunocytochemistry in S2 cells treated with different combinations of 455 siRNAs, as indicated. Green, H3K9me3 signals; blue, DAPI. Scale bar, $2 \mu \mathrm{m}$.(D) Violin plot for

456 fold-change of $\mathrm{H} 3 \mathrm{~K} 9 \mathrm{me} 3, \mathrm{H} 3 \mathrm{~K} 4 \mathrm{me} 3$ and $\mathrm{H} 3 \mathrm{~K} 27 \mathrm{me} 3$ signals on CHARRs-targeted peaks in 457 response to Dicer-2 knockdown. KD: knockdown. (E) H3K9me3 ChIP-seq signals on 458 representative genomic loci, one corresponding to a CHARR-target locus (left) and a non459 CHARR-target locus (right) in response to Dicer-2 knockdown, complemented with either 460 scrambled or CHARRs-derived siRNAs. ChIP-seq signals for euchromatin (H3K4me3) or 461 facultative heterochromatin (H3K27me3) were shown for comparison.

\section{Rescuing global heterochromatin defects in Dicer-2 deficient cells}

471 (see first two tracks in Fig. 6E), suggesting that Dicer-2 is functionally required for maintaining

472 heterochromatin genome-wide.

474 heterochromatin defects, and if so, whether the rescue required their targeting specificity. Because

475 those siRNA mimics were designed to target 10 representative CHARRs (Supplementary Table

476 3), we thus analyzed the H3K9me3 ChIP-seq signals on $1 \mathrm{~kb}$-binned genomic regions that show 
477 homology with at least one of the CHARR-derived siRNA mimics (Fig. 6F) in comparison with

478 genomic regions that showed H3K9me3 ChIP-seq signals but with $<50 \%$ homology with any of

479 those CHARR-derived siRNA mimics (Fig. 6G). Indeed, we found that CHARR-derived siRNA

480 mimics, but not scrambled siRNA, effectively rescued H3K9me3 ChIP-seq signals on CHARRs

481 target genomic regions (compare lane 2 vs. 4 in Fig. 6F), but modest at best (likely due to a

482 remaining degree of heterotropic interactions) on none CHARRs target regions (compare lane 2

483 vs. 4, p=0.054, in Fig. 6G). As expected, little H3K4me3 and H3K27me3 ChIP-seq signals were

484 detected in $\mathrm{H} 3 \mathrm{~K} 9 \mathrm{me} 3$-marked genomic loci. These general trends were also illustrated on two

485 representative genome loci for CHARRs targets (Fig. 6E, left and Supplementary Fig. 9A) and

486 none CHARRs targets (Fig. 6E, right and Supplementary Fig. 9B). Collectively, these data

487 demonstrated that a pool of trans-acting CHARR-derived siRNA mimics was able to bypass the

488 functional requirement of Dicer-2 for maintaining heterochromatin homeostasis on their target

489 regions in Drosophila S2 cells.

491 CHARR-derived endo-siRNAs required for faithful chromosome segregation

492 It has been previously documented that knockdown of Dicer-2 and Ago-2 caused

493 significant defects in chromosome segregation during cell division in S2 cells(Pek and Kai 2011).

494 Because Dicer-2 and Ago-2 are key siRNA pathway components, it is reasonable to extrapolate

495 that endo-siRNAs processed by Dicer-2 and loaded on Ago-2 are responsible for the phenotype,

496 but direct evidence for this critical conclusion has been lacking. Given that the CHARRs we 
498 genome (see Fig. 3B) and most of these CHARRs-derived were bound by Ago-2 (see

499 Supplementary Fig. 7C), we took advantage of the ability of CHARRs-derived siRNAs to rescue

500 most heterochromatin defects to ask whether these siRNA mimics were also able to rescue the

501 chromosome segregation defects. To this end, we first confirmed that knockdown of either Dicer-

5022 or Ago-2 caused cell cycle defects, and as expected, we detected G1-S arrest in both cases (Fig.

503 7A, B and Supplementary Fig. 10). Importantly, we found that CHARRs-derived siRNAs, but not

504 scrambled siRNA, were able to rescue the cell cycle defects in Dicer-2 knockdown cells (Fig. 7A),

505 but not Ago-2 knockdown cells (Fig. 7B). These data are fully in line with the requirement of

506 Dicer-2 for processing CHARRs into small endo-siRNAs, which could be bypassed by the

507 transfected siRNA mimics, although these siRNA mimics would still need Ago-2 to execute their

508 functions in the cell.

We closely examined the mitotic defects in Dicer-2 knockdown cells, noting both mis-

510 aligned chromosomes and lagged as well as mis-segregated chromosomes (Fig. 7C and

511 Supplementary Fig. 11A) in about equal frequencies (Fig. 7D). Importantly, transfection of

512 CHARRs-derived siRNA mimics into these Dicer-2 deficient cells, but not scrambled siRNA,

513 were sufficient to rescue these defects (Fig. 7D and Supplementary Fig. 11A). In contrast, we

514 observed similar mitotic defects in Ago-2 knockdown cells, but the CHARRs-derived siRNAs

515 failed to correct the phenotype (Fig. 7E and Supplementary Fig. 11B). Taken together, these results

516 provide unequivocal evidence for active retrotransposon RNAs to preserve pericentromeric 
517 heterochromatin homeostasis and thus cell cycle progression through the Dicer-2/Ago-2 mediated

518 endo-siRNA pathway.

A

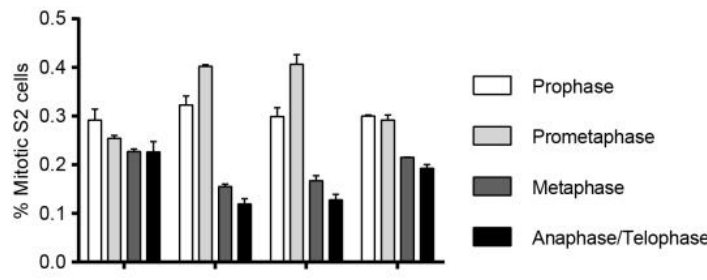

B

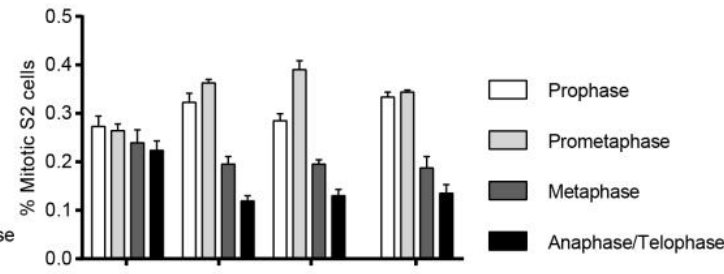

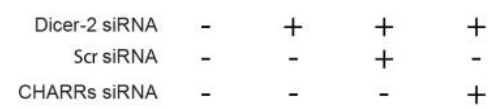

C

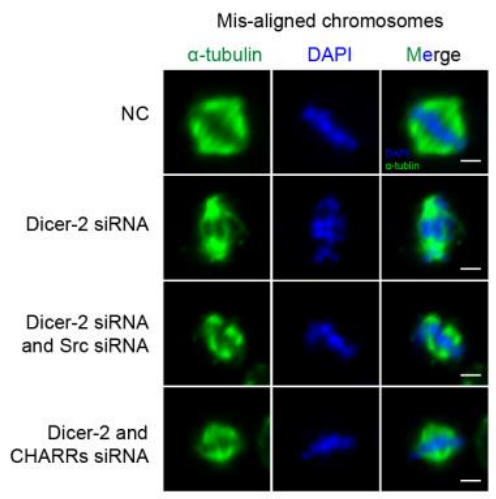

E

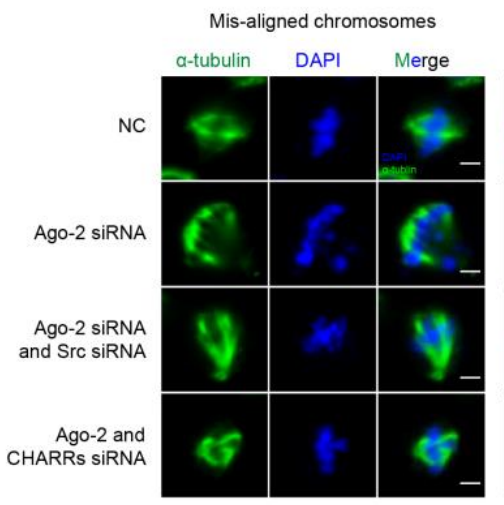

Chromosomes mis-segregation

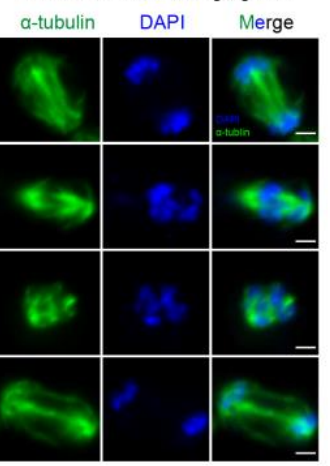

Chromosomes mis-segregation

a-tubulin DAPI Merge

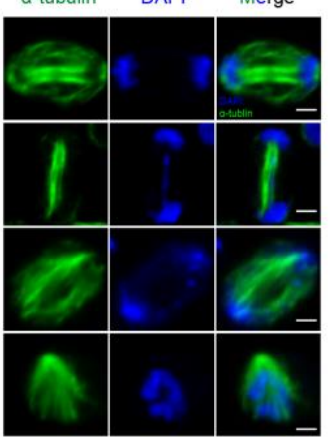

D
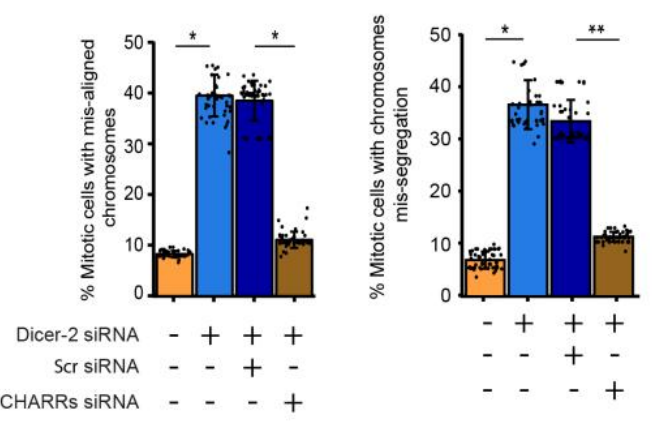

$\mathrm{F}$

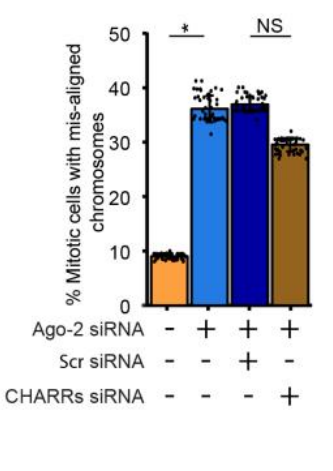
cell division defects

522 (A and B) Percentages of S2 cells at each stage of mitosis in response to knockdown of Dicer-2 523 (A) or Ago-2 (B) and treatment with either scrambled or CHARRs-derived siRNAs. $\mathrm{n}=50$ for each 
524 experiment. (C) Dicer-2 knockdown-induced chromosomes mis-alignment (left) and mis525 segregation (right) and rescue by CHARRs-derived siRNAs, but not scrambled siRNA. Green, 526 stained $\alpha$-tubulin; blue, DAPI. Scale bar, $2 \mu \mathrm{m}$.(D) Percentages of S2 cells at metaphase exhibiting 527 mis-aligned chromosomes (left) and anaphase exhibiting lagging chromosomes (right) at different 528 experimental conditions. $\mathrm{n}=50$ for each condition. $* \mathrm{p}<0.05$, ** $\mathrm{p}<0.01$, NS: not significant 529 (multiple group Student's t test). (E and F) Similar to C and D except on Ago-2 knockdown cells. $530 \mathrm{n}=50$ for each condition. ${ }^{*} \mathrm{p}<0.05,{ }^{*} \mathrm{p}<0.01$, NS: not significant (multiple group Student's $\mathrm{t}$ test).

\section{DISCUSSION} prohibitive to transcription, mounting evidence suggests that transcription is actually required to

538 initiate heterochromatin formation. This so-called nascent RNA model(Buhler et al. 2006; Holoch

542 no shortage of repeat-derived RNAs to help maintain heterochromatin. The central question is

543 what is the nature of these RNAs and where they come from? 
547 attacked this fundamental problem by using the newly elucidated RNA-DNA interactome in

548 Drosophila somatic cells(Li et al. 2017). Taking advantage of such high density interactome, we

549 now show that various active retrotransposons, especially those from the Gypsy family of the LTR

550 class, produce a large amount of repeat RNAs that are selectively associated with pericentromeric

551 regions in the fly genome. Interestingly, while a subset of those repeat-derived RNAs tends to act

552 in cis, another subset appears to interact with DNA in both cis and trans modes. Particularly

553 interesting is a general trend we have uncovered, where DNA loci with more active local

554 transcription seem to inversely correlate to their ability to attract trans-acting RNAs from related

555 repeat species. Therefore, RNAs released from those more active loci may supply extra RNAs to

556 act in trans on less transcribed loci. This "community" act of repeat-derived RNAs may thus help

557 "patch up" certain heterochromatic regions "damaged" by chromatin remodeling activities in the

558 cell, such as that catalyzed by the H3K9me3 demethylase, thereby ensuring heterochromatin

559 homeostasis for stable epigenetic inheritance.

560 In fission yeast, repeat-derived RNAs are amplified by an RNA-dependent RNA

561 polymerase, which may supply a population of trans-acting RNAs to help initiate and/or maintain

562 heterochromatin in other genomic loci(Yu et al. 2018). A different mechanism is employed in

563 Drosophila germline where repeat-rich transcripts are processed and amplified by the piRNA

564 machinery to ensure efficient silencing of retrotransposons to protect the genome integrity as well

565 as to help maintain heterochromatin in centromeric and pericentromeric regions to ensure accurate

566 chromosome segregation during cell division in gonad(Muerdter et al. 2013; Iwasaki et al. 2015). 
567 The problem is that such RNA amplification mechanism does not seem to exist in somatic cells of

568 flies and mammals. The endo-siRNA pathway has clearly been implicated in Drosophila somatic

569 cells(Czech et al. 2008; Ghildiyal et al. 2008), although it remains unclear whether such endo-

570 siRNA pathway also operates in somatic cells of mammals, which might result from less attention

571 paid on processing of repeat-derived RNAs by the conserved siRNA machinery known to be

572 highly active in mammals. In any case, at least in Drosophila S2 cells, we now document that such

573 endo-siRNA pathway is responsible for generating small RNAs that can act in cis and trans to help

574 maintain heterochromatin homeostasis in pericentromeric regions. We envision a similar

575 mechanism that may also operate in somatic cells in mammals, which requires future investigation

576 once the RNA-DNA interactome of much higher density becomes available.

578 to cause mitotic defects in both fly and mammalian cells(Pek and Kai 2011; Huang et al. 2015),

579 but it has been unclear whether these small RNA machineries act through their traditional functions

580 or through some new mechanism(s) in the nucleus. We now show that a pool of synthetic repeat-

581 derived siRNA mimics is able to rescue all measurable cell cycle defects in Drosophila S2 cells,

582 which is fully compatible to the central role of the endo-siRNA pathway in ensuring cell cycle

583 progression through maintaining pericentromeric heterochromatin. We speculate the conversed

584 function of this pathway in mammals, although different classes of retrotransposons are likely

585 involved in different organisms. 
587 genetic inheritance in somatic cells suggests a key and immediate benefit of active retrotransposons for the genome. This realization is interesting because retrotransposons have been traditionally viewed as mutagens in the genome, although their relatively random actions may

590 facilitate genome evolution in the long run. In Drosophila germ cells, an RNA amplification

591 mechanism has been evolved to maximally suppress this mutagen function of retrotransposons to

592 ensure genome integrity. We now show that despite the lack of such RNA amplification

593 mechanism in somatic cells, the endo-siRNA machinery is still quite active to ensure the supply

594 of repeat-derived small RNAs. Therefore, without worrying about the responsibility to transmit

595 genetic materials to offspring, somatic cells may tolerate potential genome vulnerability in

596 exchange for stable genetic inheritance during development and differentiation. As higher

597 eukaryotic genomes are populated with enormous amounts of repeat sequences, we suggest that

598 some of those "junk" DNA sequences actually have important functions while most others are

599 fossil of genome evolution.

METHODS

605 S2 cells were cultured under sterile conditions at $26^{\circ} \mathrm{C}$ in Schneider medium (Invitrogen) 606 containing $10 \%$ heat-inactivated fetal bovine serum and $100 \mu \mathrm{g} / \mathrm{ml}$ penicillin-streptomycin. S2 cell 607 lines are negative for mycoplasma contamination. 


\section{Alignment of GRID-seq reads to the Drosophila genome}

GRID-seq raw reads were split into RNA and DNA reads according to the designed bivalent linker. Trimmomatic(Bolger et al. 2014) was used to remove adapter sequences and filter low-quality reads by using the parameters MINLEN: 18 and SLIDINGWINDOW: 2:20. Filtered reads were aligned to the Drosophila genome (genome version :dm6) with ShortStack(Axtell 2013) using the parameter $-m 400$ for DNA reads and $-m 200$ for RNA reads, respectively. Multiple mapped reads were weighted based on the frequencies of neighbor uniquely mapped reads (Supplementary Fig. 1C). Unmapped reads were cleaned with SAMtools(Li et al. 2009) using the parameter $-F 4$, and the weighted score of each read was recorded in the fifth column of bed file.

\section{Annotation of RNA and DNA reads}

620 Annotated unique RNAs of Drosophila was downloaded from FlyBase(Drysdale 2008) and the repeat sequences from RepeatMasker track in the UCSC genome browser(Jurka et al. 2005). RNA reads were annotated to unique and repeat RNAs using IntersectBed tool with the parameter $-f$ 1.0, -split and $-s$. The Drosophila genome was scanned with EMBOSS (Rice et al. 2000) based on Alu I restriction sites from REBASE (Roberts et al. 2015) to generate AluI DNA bins. IntersectBed tool was used to connect Alu I DNA bins using the parameter $-f$ 1.0.

\section{Assigning RNA-DNA interactions}

628 Multiple read pairs that have the same mapped RNA and DNA loci associated with the same PCR primer sequences were considered PCR duplicated, and thus were counted only once. The following equation was used to compute RNA-DNA interactions in each AluI DNA bin:

\section{2}

Where $n$ is the number of assigned read pairs. For each read pair $i, R_{i}$ is the contribution score of this read to the RNA part and $D_{i}$ is the contribution score of this read to the interacting AluI DNA 634 bin. $R_{i}=1$, if the RNA part of the read pair is uniquely mapped, or equals to a fraction based on the weighted score.

$$
\operatorname{Num}_{(\text {interaction })}=\sum_{i=1}^{n}\left(R_{i} * D_{i}\right)
$$

638 A mixed GRID-seq library from human MDA-MB-231 and Drosophila S2 cells was used to 639 construct the non-specific RNA-DNA interaction profile. RNA reads, which were only mapped to 640 the human genome (hg38) using Bowtie(Langmead et al. 2009) with the parameter $-n 0$, were kept. 641 Their mated DNA reads were processed using ShortStack, as described above. The human RNA 642 signals within each $1 \mathrm{~kb}$ DNA bin were normalized to one million, which was further smoothed by 643 a moving window that includes 5 upstream and 5 downstream bins. The final coverage of the $1 \mathrm{~kb}$ 644 DNA bin $i$ is: 


$$
\operatorname{Cov}_{i}=\frac{1}{11} \sum_{i=5}^{i+5}\left(\frac{10^{6} * \sum_{m} \operatorname{Read}_{i m}}{N}\right)
$$

where $m$ is the number of reads mapped to the $1 \mathrm{k}$ DNA bin $i$ and $N$ is the total read number mapped to the Drosophila genome.

To make DNA binding scores comparable between Alu I binned versus $1 \mathrm{~kb}$ binned genome, RNA binding signals in each $1 \mathrm{k}$ DNA bin were converted to RNA binding signals in each AluI DNA bin by first dividing each $1 \mathrm{~kb}$ DNA bin into 1000 lbp bins to calculate the signal in each small bin based on $\frac{\operatorname{Cov}_{\text {bin }}}{1000}$. IntersectBed tool with the parameter $-f 1.0$ was used to compute signals in each Alu I DNA bin by summing the signals from all 1bp bins in the fragment. Finally, signals in AluI DNA bins were all normalized to signals per $1 \mathrm{~kb}$ :

where $L e n_{j}$ is the length of the AluI DNA bin $j$.

$$
\text { Score }_{\text {AluI DNA bin } j \text { in mixed library }}=\left(10^{3} * \sum_{m=1}^{L e n_{j}} \operatorname{Cov}_{m}\right) / \text { Len }_{j}
$$

\section{Filtering singular and background to identify specific RNA-DNA interactions}

659 To support specific RNA-DNA interactions, we required at least two RNA-DNA mates for each RNA transcript in a given Alu I DNA bin. We also developed two background models to simulate Poisson distribution of RNA binding signals on DNA. The first was based on uniform distribution of individual RNAs on DNA, based on which we estimated the background score:

$$
\text { Score }_{\text {interaction } i-j}=\left(\operatorname{Len}_{j} * N_{i}\right) / \text { Len }_{g}
$$

where the $L e n_{g}$ is the length of the genome, $L e n_{j}$ is the length of AluI DNA bin $j$ and $N_{i}$ is total signals for RNA $i$. This score was further normalized according to length in each AluI DNA bin, and the resulting RPK (reads per kilobase) was used as the $\lambda_{B G_{-} i}$ value to obtain the Poisson distribution of this RNA-engaged genomic interactions and to calculate the p-value for such interactions. The ratio of Num $_{\text {interaction } i-j}$ over Score $_{\text {interaction } i-j}$ was reported as the fold_change (FC) above the background. We also developed a second background model with that data deduced with RNA signals from the mixed library. We first calculated the non-specific interaction score $\left(\right.$ Score $_{A l u I}$ DNA bin $j$ in mix library $)$ based on human-derived RNA binding signals, and then used this score as the $\lambda_{B G_{-} \text {mix }}$ value to obtain the Poisson distribution of human RNAengaged genomic interactions and to calculate the $\mathrm{p}$-value for such interactions. The ratio of length and sequencing depth normalized $N u m_{\text {interaction } i-j}$ (in RPKM) over $\lambda_{B G_{-} \text {mix }}$ was reported as the fold_change (FC). RNA-DNA interactions that met the requirement of p-value $<0.05$ and the fold_change (FC) $>2$ based on both background models were considered specific and thus retained 
679 Data normalization for comparison between GRID-seq libraries and different RNAs within 680 the same libraries

681 The interaction RNA-DNA score for each RNA is affected by the sequencing depth in different 682 GRID-seq libraries, the length of each Alu I DNA bin, and the length of each RNA. To enable 683 comparison among different libraries and different RNAs within the same libraries, we normalized 684 these variables according to:

$$
\operatorname{RRPKM}_{i j}=\left(\mathrm{Num}_{i j} * 10^{6} * 10^{3} * 10^{3}\right) /\left(\text { TotalReadCounts } * \text { Len_D } D_{j} * \text { Len_R } R_{i}\right)
$$

where $N_{u m}$ is the interaction score of RNA $i$ with Alu I DNA bin $j$, TotalReadCounts is the sequencing depth of individual libraries, $L e n_{-} D_{j}$ is the length of Alu I DNA bin $j$ and $L e n_{-} R_{i}$ is the length of RNA $i$.

\section{Comparison between GRID-seq and ChAR-seq datasets}

692 ChAR-seq raw data were downloaded from the GEO database (Supplementary Table 2). PCR duplicates were removed using Clumpify with default parameters. All five independent ChAR-seq libraries were combined followed by adapter trimming and filtering low-quality reads with Trimmomatic using the parameters MINLEN: 36, LEADING:3 TRAILING:3 and SLIDINGWINDOW: 4:15. Filtered ChAR-Seq reads were split into paired RNA and DNA reads, and then processed as described above for the GRID-seq data. FeatureCounts was used to calculate reads in $1 \mathrm{~kb}$ DNA bins and then converted to RPKM using edgeR package in R language. For validation for assigning multi-mapped reads, we only considered $1 \mathrm{~kb}$ DNA bins that contain newly assigned multi-mapped reads from the GRID-seq dataset.

\section{Comparison of RNA-DNA interactions in relationship with chromatin marks} processing were similar to the procedures for processing the GRID-seq data with parameters adjusted to MINLEN: 36 and SLIDINGWINDOW: 4:20 according to the read length. Filtered reads were mapped to the reference Drosophila genome (dm6) using STAR (Dobin et al. 2013) with the parameters: --outFilterScoreMinOverLread 0.1, -outFilterMatchNminOverLread 0.1, -alignIntronMax 1 and --alignEndsType EndToEnd. The wig files of each chromatin mark ChIPseq dataset was obtained through STAR using the parameters:-outWigTypewiggle read1_5p, -outWigStrandUnstranded and --outWigNormRPM. Enriched peaks were detected by MACS2 with input data as control. Top 500 peaks were used for further analysis.

\section{Identification of repeat RNA on constitutive heterochromatin}

715 The ChIP-seq data for constitutive heterochromatin markers (H3K9me3 and HP1) were obtained 716 from the GEO database (Supplementary Table 2) and the RPKM values were calculated on 10kb 
DNA bins. The Pearson correlation score was calculated on each DNA bin containing binding signals from repeat RNAs. Repeat sequences, except rRNAs, were defined as CHARRs if the correlation score with H3K9me3 or HP1 is $>0.3$.

\section{Analysis of CHARR-DNA interactions relative to Hi-C defined compartments} Public Hi-C data from S2 cells were downloaded (Supplementary Table 2) and processed with Trimmomatic using default settings plus trim tool in Homer using the parameter -3 AAGCTT. Trimmed reads with length of $\geq 38 \mathrm{nt}$ were mapped to the Drosophila genome using end-to-end alignment model provided by Bowtie2 (Ref (Langmead and Salzberg 2012)). We discarded potential PCR duplicates as well as reads with no useful information, including (1) read pairs separated $<1.5 \times$ of the sequenced insert fragment length, (2) reads from $10 \mathrm{~kb}$ regions containing $>5 \times$ of the average coverage, (3) read pairs lacking restriction sites at the 3 ' end of either read within the estimated fragment length, (4) reads with their ends resulting from selfligation with adjacent restriction fragments. We then used the filtered dataset to perform PCA analysis with HOMER, using runHiCpca.pl with the parameters - res $10 \mathrm{~kb}$ and -superRes $20 \mathrm{~kb}$ and compartments analysis using runHiCpca.pl and findHiCCompartments.pl. Finally, CHARRDNA interactions signals and Hi-C compartments were intersected with IntersectBed from BedTools. The length of Hi-C compartment A and B was normalized to $1 \mathrm{Mb}$. processed as with the ChIP-seq data for chromatin marks. Annotated unique and repeat RNA species were used to calculate individual transcription scores by using FeatureCounts and EdgeR.

Small RNA-seq data were similarly processed as above using adjusted Trimmomatic parameters: ribosomal RNAs with default setting. We also used ShortStack to map both uniquely and multimapped reads to obtain the RPM value for each CHARR.

Reads for the downloaded Ago2 RIP data were blasted to identify repeat RNA-derived sequences using the parameters -outfmt 6 and-word_size 7. Reads will be kept for further analysis if the mismatch number is under 3 and the mapping region of the reads equals to the whole length of the reads. We then counted the reads number for each repeat sequence.

\section{siRNAs-mediated knockdown of Dicer-2 and Ago-2 and immunofluorescence}

753

Transfection was performed, as described(Rogers and Rogers 2008), using the "bathing" method

754 for siRNA delivery. Cells were counted, pelleted and resuspend at $1-5 \times 10^{6}$ cells $/ \mathrm{ml}$ in serum free media. We added $\sim 10-30 \mu \mathrm{g}$ dsRNA to each well in the 6-well tissue culture plate to obtain the final concentration of 25-50 nM. About $1 \mathrm{ml}$ of cells were seeded each well in the 6-well plate and 
incubate at room temperature for 30 min followed by the addition of $3 \mathrm{ml}$ complete media with $10 \%$ FBS to each well. This process was repeated every other day for three times before harvesting the cells for downstream assays. Sequences of individual siRNAs were listed in Supplementary Table 3.

For immunostaining, S2 cells were washed in $1 \times \mathrm{PBS}$ and fixed in 4\% paraformaldehyde (pH-7.2) for $10 \mathrm{~min}$ at room temperature. After washing four times, cells were permeabilized with $0.1 \%$ Triton X100 in 1× PBS for $5 \mathrm{~min}$ at room temperature. Permeabilized cells were then incubated in $1 \%$ normal goat serum in $1 \times$ PBST for $30 \mathrm{~min}$ at room temperature, and then with the primary antibody ( $\alpha$-rabbit H3K9me3 1:500) and secondary antibodies (1:400) both in blocking buffer (3\% BSA, $1 \%$ goat serum in PBST), each for $1 \mathrm{~h}$ at room temperature. Cells were washed three times at room temperature, each for 5 min with $1 \times$ PBST. After mounting on coverslip with DAPI, cells were examined under Zeiss LSM-700 confocal laser scanning microscope.

\section{ChIP-seq library construction and data analysis} DNA libraries were constructed using the NEBNext ${ }^{\circledR}$ Ultra ${ }^{\mathrm{TM}}$ II DNA Library Prep kit (NEB, USA) following manufacturer's recommendations. After end repair, 5' phosphorylation. and dAtailing of purified DNA fragments, NEBNext adaptors with hairpin loop structure were ligated and library fragments were purified with SPRIselect sample purification beads (NEB, USA). After ensuring the quality of libraries on the Agilent Bioanalyzer 2100 system, individual libraries were sequenced on the Illumina HiSeq X Ten platform to generate $150 \mathrm{bp}$ paired-end.

Adapters and low-quality reads were filtered to obtain clean reads and clean reads mapped to the reference Drosophila genome (dm6) using Bowtie2. The wig files of each chromatin mark ChIPseq dataset was obtained by using bamCoverage tools from Deeptools(Ramirez et al. 2016) with the parameters: --binSize 1000, --normalizeTo1x 142573017 and--ignoreForNormalization chrM. Enriched peaks were detected by MACS2 with input data as control using the parameters $-f$ BAMPE --nomodel --keep-dup all --broad.

\section{Analysis of mitotic defects}

Medium and fetal bovine serum were batch tested for support of normal cell growth and RNAi efficiency. For the knockdown group, specific synthetic siRNA was added to cell culture in 24well plates. After siRNA treatment for 4 days, cells were resuspended and transferred to glassbottom, 24-well plates (Cellvis) and allowed to adhere for $2.5 \mathrm{hrs}$ before fixation, as described(Goshima et al. 2007). Cells were fixed in $4 \%$ paraformaldehyde for $10 \mathrm{~min}$, permeabilized with $0.1 \%$ Triton X100 in PBS for $5 \mathrm{~min}$ and incubated overnight at $4^{\circ} \mathrm{C}$ with anti-

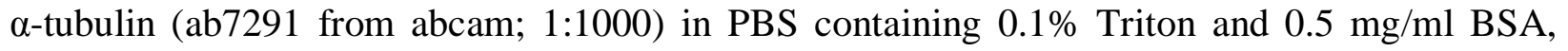
followed by staining with secondary antibodies and DAPI $(1 \mu \mathrm{g} / \mathrm{ml})$. For the rescue group, siRNAi treatment was performed as above followed by transfection of CHARRs siRNA 2 days later. 
795 Immunostained specimens were imaged under a Zeiss LSM-700 confocal laser scanning

796 microscope, using a 63x 1.4 NA oil immersion objective to achieve high resolution. We imaged 797 two channels (DAPI, AF488) at typically 10-20 sites/well to obtain 50 metaphase cells/well on 798 average.

\section{Western blotting, RT-qPCR and Northern blotting}

801 Total cell lysate containing 15-25 $\mu \mathrm{g}$ protein from S2 cell cultures were fractionated by SDS802 PAGE, immunoblotted and probed with specific antibodies. After incubation with peroxidase803 conjugated secondary antibodies (1:5000; Abcam), blots were developed with Supersignal West 804 Pico Chemiluminescent Substrate (Pierce) and exposed to film (SAGECREATION, MiNiChemi). 805 Signal intensity for Dicer-2, H3K9me3, H3K27me3, H3K4me3 and Tublin was quantified using 806 ImageJ.

808 For RNA quantification, total RNA was extracted, and genomic DNA was removed with DNase I 809 (Roche, 04716728001). First-strand cDNA was generated with the SuperScript III Fist-Stand using 810 random hexamers. The expression levels of RNAs were quantified on Rotor-Gene Q (QIAGEN) 811 and normalized against GAPDH mRNA. PCR primers sequences were listed in Supplementary 812 Table 3.

About $30 \mu \mathrm{g}$ of total RNA isolated with Trizol was loaded into each lane of agarose gel and blotted onto membrane with Chemiluminescent Nucleic Acid Detection Module (Thermo Fisher) according to manufacturer's instruction. RNA probes were labeled by in vitro transcription of plasmids with T7 RNA polymerase (Promega) in the presence of Biotin RNA labeling mix (Roche). The primers used were listed in Supplementary Table 3.

\section{Quantification and statistical analysis}

821 Statistical parameters were reported either in individual figures or corresponding figure legends.

822 Quantified data were in general presented as bar/line plots, with the error bar representing mean \pm 823 SEM, or boxplot showing the median (middle line), first and third quartiles (box boundaries), and 824 furthest observation or 1.5 times of the interquartile (end of whisker). All statistical analyses were 825 done in $\mathrm{R}$. Wherever asterisks are used to indicate the statistical significances, *stands for $\mathrm{p}<0.05$; $826 * *$ for $\mathrm{p}<0.01$, *** for $\mathrm{p}<0.001$ and NS for not significant.

\section{Data Access} All sequencing and processed files were deposited to Gene Expression Omnibus under accession 831 https://www.ncbi.nlm.nih.gov/geo/query/acc.cgi?acc=GSE134307, then enter token 832 qzexqwwmbbujhol into the box. All software and the datasets used in this study are available from 833 the corresponding author, Xiang-Dong Fu (xdfu@ucsd.edu), upon reasonable request. 


\section{ACKNOWLEDGEMENTS}

We thank Dr. Yang Yu for providing S2 cells. This work was supported by grants from the National Key R\&D Program of China (2016YFC0901702, 2016YFC09010002) and the National Natural Science Foundation of China (31871294, 31520103905) to R.S.C. and S.M.H., and NIH grants (HG004659, GM049369 and GM052872) to X.D.F.

\section{AUTHOR CONTRIBUTIONS}

Conceptualization, X.D.F., R.S.C., and S.M.H.; Methodology development and data analysis, Y.J.H.; Generation of the GRID-seq data in S2 cells, X.L.; Experimental design and execution, Y.J.H., D.P.W. and S.H.W.; Data interpretation and discussion, Y.J.H., X.D.F., R.S.C., S.M.H., D.P.W, S.H.W, P.Z., J.Y.C., C.W.S., and D.H.L.; Paper writing, Y.J.H. and X.D.F.

\section{DISCLOSURE DECLARATION}

The authors declare no competing interests.

\section{REFERENCES}

Allshire RC, Nimmo ER, Ekwall K, Javerzat JP, Cranston G. 1995. Mutations derepressing silent centromeric domains in fission yeast disrupt chromosome segregation. Genes Dev 9: 218-233.

Avner P, Heard E. 2001. X-chromosome inactivation: counting, choice and initiation. Nat Rev

Axtell MJ. 2013. ShortStack: comprehensive annotation and quantification of small RNA genes. Rna 19: 740-751.

Baker BS, Gorman M, Marin I. 1994. Dosage compensation in Drosophila. Annu Rev Genet 28: 491-521.

Bell JC, Jukam D, Teran NA, Risca VI, Smith OK, Johnson WL, Skotheim JM, Greenleaf WJ, Straight AF. 2018. Chromatin-associated RNA sequencing (ChAR-seq) maps genomewide RNA-to-DNA contacts. Elife 7.

Bolger AM, Lohse M, Usadel B. 2014. Trimmomatic: a flexible trimmer for Illumina sequence data. Bioinformatics 30: 2114-2120. 
862

863

864

865

866

867

868

869

870

871

872

873

874

875

876

877

878

879

880

Buhler M, Verdel A, Moazed D. 2006. Tethering RITS to a nascent transcript initiates RNAiand heterochromatin-dependent gene silencing. Cell 125: 873-886.

Czech B, Malone CD, Zhou R, Stark A, Schlingeheyde C, Dus M, Perrimon N, Kellis M, Wohlschlegel JA, Sachidanandam R et al. 2008. An endogenous small interfering RNA pathway in Drosophila. Nature 453: 798-802.

Dobin A, Davis CA, Schlesinger F, Drenkow J, Zaleski C, Jha S, Batut P, Chaisson M, Gingeras TR. 2013. STAR: ultrafast universal RNA-seq aligner. Bioinformatics 29: 15-21.

Drysdale R. 2008. FlyBase : a database for the Drosophila research community. Methods in molecular biology (Clifton, NJ) 420: 45-59.

Ekwall K, Javerzat JP, Lorentz A, Schmidt H, Cranston G, Allshire R. 1995. The Chromodomain Protein Swi6 - a Key Component at Fission Yeast Centromeres. Science 269: 1429-1431.

Fagegaltier D, Bouge AL, Berry B, Poisot E, Sismeiro O, Coppee JY, Theodore L, Voinnet O, Antoniewski C. 2009. The endogenous siRNA pathway is involved in heterochromatin formation in Drosophila. Proceedings of the National Academy of Sciences of the United States of America 106: 21258-21263.

Franke A, Baker BS. 1999. The rox 1 and rox 2 RNAs are essential components of the compensasome, which mediates dosage compensation in Drosophila. Molecular Cell 4: $117-122$.

Ghildiyal M, Seitz H, Horwich MD, Li C, Du T, Lee S, Xu J, Kittler EL, Zapp ML, Weng Z et al. 2008. Endogenous siRNAs derived from transposons and mRNAs in Drosophila somatic cells. Science 320: 1077-1081.

Goshima G, Wollman R, Goodwin SS, Zhang N, Scholey JM, Vale RD, Stuurman N. 2007. Genes required for mitotic spindle assembly in Drosophila S2 cells. Science 316: 417421.

Grewal SI, Jia S. 2007. Heterochromatin revisited. Nat Rev Genet 8: 35-46.

Grewal SI, Klar AJ. 1997. A recombinationally repressed region between mat 2 and mat 3 loci shares homology to centromeric repeats and regulates directionality of mating-type switching in fission yeast. Genetics 146: 1221-1238.

Halic M, Moazed D. 2009. Transposon silencing by piRNAs. Cell 138: 1058-1060. 
Hall LL, Carone DM, Gomez AV, Kolpa HJ, Byron M, Mehta N, Fackelmayer FO, Lawrence JB. 2014. Stable C0T-1 repeat RNA is abundant and is associated with euchromatic interphase chromosomes. Cell 156: 907-919.

Holoch D, Moazed D. 2015a. RNA-mediated epigenetic regulation of gene expression. Nat Rev Genet 16: 71-84.

Holoch D, Moazed D. 2015b. Small-RNA loading licenses Argonaute for assembly into a transcriptional silencing complex. Nat Struct Mol Biol 22: 328-335.

Huang C, Wang X, Liu X, Cao S, Shan G. 2015. RNAi pathway participates in chromosome segregation in mammalian cells. Cell Discov 1: 15029.

Iwasaki YW, Siomi MC, Siomi H. 2015. PIWI-Interacting RNA: Its Biogenesis and Functions. Annu Rev Biochem 84: 405-433.

Jurka J, Kapitonov VV, Pavlicek A, Klonowski P, Kohany O, Walichiewicz J. 2005. Repbase Update, a database of eukaryotic repetitive elements. Cytogenetic and genome research 110: $462-467$.

Kandasamy SK, Fukunaga R. 2016. Phosphate-binding pocket in Dicer-2 PAZ domain for high-

Kellum R, Alberts BM. 1995. Heterochromatin Protein-1 Is Required for Correct Chromosome Segregation in Drosophila Embryos. J Cell Sci 108: 1419-1431.

Kopylova E, Noe L, Touzet H. 2012. SortMeRNA: fast and accurate filtering of ribosomal RNAs in metatranscriptomic data. Bioinformatics 28: 3211-3217.

Langmead B, Salzberg SL. 2012. Fast gapped-read alignment with Bowtie 2. Nature methods 9: 357-359.

Langmead B, Trapnell C, Pop M, Salzberg SL. 2009. Ultrafast and memory-efficient alignment

915 Lee YS, Nakahara K, Pham JW, Kim K, He Z, Sontheimer EJ, Carthew RW. 2004. Distinct roles 916 for Drosophila Dicer-1 and Dicer-2 in the siRNA/miRNA silencing pathways. Cell 117: 917 69-81.

918 Li H, Handsaker B, Wysoker A, Fennell T, Ruan J, Homer N, Marth G, Abecasis G, Durbin R. 919 2009. The Sequence Alignment/Map format and SAMtools. Bioinformatics 25: 20782079. 
921 Li X, Zhou B, Chen L, Gou LT, Li H, Fu XD. 2017. GRID-seq reveals the global RNAchromatin interactome. Nat Biotechnol 35: 940-950.

923 Lippman Z, Gendrel AV, Black M, Vaughn MW, Dedhia N, McCombie WR, Lavine K, Mittal V, May B, Kasschau KD et al. 2004. Role of transposable elements in heterochromatin and epigenetic control. Nature 430: 471-476.

Muerdter F, Guzzardo PM, Gillis J, Luo Y, Yu Y, Chen C, Fekete R, Hannon GJ. 2013. A genome-wide RNAi screen draws a genetic framework for transposon control and primary piRNA biogenesis in Drosophila. Mol Cell 50: 736-748.

Pek JW, Kai T. 2011. DEAD-box RNA helicase Belle/DDX3 and the RNA interference pathway promote mitotic chromosome segregation. Proc Natl Acad Sci U S A 108: 12007-12012.

931 Peters AH, O'Carroll D, Scherthan H, Mechtler K, Sauer S, Schofer C, Weipoltshammer K, Pagani M, Lachner M, Kohlmaier A et al. 2001. Loss of the Suv39h histone

Ramirez F, Ryan DP, Gruning B, Bhardwaj V, Kilpert F, Richter AS, Heyne S, Dundar F, Manke T. 2016. deepTools2: a next generation web server for deep-sequencing data analysis. Nucleic Acids Res 44: W160-165.

944 Roberts RJ, Vincze T, Posfai J, Macelis D. 2015. REBASE--a database for DNA restriction and 945 modification: enzymes, genes and genomes. Nucleic Acids Res 43: D298-299.

946 Rogers SL, Rogers GC. 2008. Culture of Drosophila S2 cells and their use for RNAi-mediated 947 loss-of-function studies and immunofluorescence microscopy. Nature protocols 3: 606948611.

949 Sridhar B, Rivas-Astroza M, Nguyen TC, Chen W, Yan Z, Cao X, Hebert L, Zhong S. 2017. 950 Systematic Mapping of RNA-Chromatin Interactions In Vivo. Curr Biol 27: 610-612. 
951 Stein P, Svoboda P, Anger M, Schultz RM. 2003. RNAi: Mammalian oocytes do it without RNA-dependent RNA polymerase. Rna 9: 187-192.

953 Sun FL, Cuaycong MH, Craig CA, Wallrath LL, Locke J, Elgin SC. 2000. The fourth

954 chromosome of Drosophila melanogaster: interspersed euchromatic and heterochromatic domains. Proc Natl Acad Sci U S A 97: 5340-5345.

956 Tschiersch B, Hofmann A, Krauss V, Dorn R, Korge G, Reuter G. 1994. The protein encoded by the Drosophila position-effect variegation suppressor gene $\mathrm{Su}(\mathrm{var}) 3-9$ combines domains of antagonistic regulators of homeotic gene complexes. EMBO J 13: 3822-3831.

Vagin VV, Sigova A, Li C, Seitz H, Gvozdev V, Zamore PD. 2006. A distinct small RNA pathway silences selfish genetic elements in the germline. Science 313: 320-324.

961 Verdel A, Jia S, Gerber S, Sugiyama T, Gygi S, Grewal SI, Moazed D. 2004. RNAi-mediated targeting of heterochromatin by the RITS complex. Science 303: 672-676.

963 Volpe T, Martienssen RA. 2011. RNA interference and heterochromatin assembly. Cold Spring Harbor perspectives in biology 3: a003731.

965 Volpe TA, Kidner C, Hall IM, Teng G, Grewal SI, Martienssen RA. 2002. Regulation of heterochromatic silencing and histone H3 lysine-9 methylation by RNAi. Science 297: 1833-1837.

Yu R, Wang X, Moazed D. 2018. Epigenetic inheritance mediated by coupling of RNAi and

970 Zhang YJ, Guo L, Gonzales PK, Gendron TF, Wu Y, Jansen-West K, O'Raw AD, Pickles SR, Prudencio M, Carlomagno Y et al. 2019. Heterochromatin anomalies and doublestranded RNA accumulation underlie C9orf72 poly(PR) toxicity. Science 363. 